\title{
HOMOGENIZATION OF THIN PIEZOELECTRIC PERFORATED SHELLS
}

\author{
Marius Ghergu ${ }^{1}$, Georges Griso $^{2}$, Houari Mechkour $^{3}$ and Bernadette Miara ${ }^{4}$
}

\begin{abstract}
We rigorously establish the existence of the limit homogeneous constitutive law of a piezoelectric composite made of periodically perforated microstructures and whose reference configuration is a thin shell with fixed thickness. We deal with an extension of the Koiter shell model in which the three curvilinear coordinates of the elastic displacement field and the electric potential are coupled. By letting the size of the microstructure going to zero and by using the periodic unfolding method combined with the Korn's inequality in perforated domains, we obtain the limit model.
\end{abstract}

Mathematics Subject Classification. 74K25, 74Q05, 35B27.

Received October 21, 2006. Revised March 18, 2007.

\section{INTRODUCTION}

The homogenization method used in the modelling of periodically structured domains has been extensively developed after the pioneering work (with a mathematical point of view) of Bensoussan et al. [3]. A rigorous asymptotic study is given in Nguetseng [20], Allaire [1], Sanchez-Hubert and Sanchez-Palencia [22, 23], Cioranescu and Donato [8] and in Cioranescu and Saint-Jean Paulin [10] in the case of thin perforated domains. The approach we use in this paper differs from these quoted before and is more similar to the periodic modulation method found in [2,17]; it relies on the periodic unfolding method introduced by Cioranescu et al. [7] and recently also extended to perforated domains [9].

The Koiter's model for elastic shells combines the effects of the two tensors: the membrane tensor and the flexural tensor. An asymptotic behavior of the displacement field in case of composite or laminated elastic shells is described, for example, in Caillerie and Sanchez-Palencia [5] or Lewiński and Telega [16]. The coupling, in the Koiter model of the two-dimensional linearized change of metric and curvature tensors which present different order of derivatives of the curvilinear coordinates of the elastic displacement field gives rise to local problems of different nature than the global one; in our paper these problems can be found in equations (30)-(32) and equations (34) respectively. Another interesting extension is due to Caillerie and Sanchez-Palencia [6] when

\footnotetext{
Keywords and phrases. Computational solid mechanics, homogenization, perforations, piezoelectricity, shells.

1 Institute of Mathematics "Simion Stoilow" of the Romanian Academy, PO Box 1-764, RO-014700, Bucharest, Romania. marius.ghergu@imar.ro

2 Laboratoire Jacques-Louis Lions, Université Pierre et Marie Curie (Paris VI), 4 Place Jussieu, 75252 Paris, France.

4 Laboratoire de Modélisation et Simulation Numérique, ESIEE, 2 Boulevard Blaise Pascal, 91360 Noisy-Le-Grand, France. miarab@esiee.fr 
the size of each microstructure is comparable with its thickness; this approach will be addressed later in our framework of piezoelectric material.

In the piezoelectric effect there exists a coupling between elastic field and electric field, i.e., under an applied mechanical force a piezoelectric body undergoes a strain that produces an electric field and conversely an applied electric field produces a mechanical stress. Many cristalline materials (such as quartz, Rochelle salt) or ceramic materials (barium titanate, lead zirconate titanate) exhibit a piezoelectric behavior. Also human skin and human bone present this property but with a very low elastic-electric coupling efficiency. These materials are used as sensors or actuators, ultrasonic or shear transducers. For a more complete study of piezoelectric materials we refer the reader to Dieulesaint and Royer [12] or Ikeda [14]. The modelling of laminated three-dimensional piezoelectric composite has already been analyzed by Bourgeat et al. [4] and Mechkour [18].

Having in mind such applications as [21] (which presents a piezoelectric porous electrode used as a spatial filter sensor) or [19] (which presents a bio-material made of a piezoelectric matrix with elastic inclusions of osteoblasts and used as a micro device supposed to improve the bone regeneration) our purpose in this paper is to combine the different previous features: shell structure, perforations and piezoelectric model to mathematically analyze a well adapted model. Hence we study the homogenization of a thin shell having periodically distributed microstructures with small size $\varepsilon$, each microstructure containing a hole. The shell is assumed to be of constant thickness and periodicity, in this case, is viewed as periodicity with respect to the curvilinear parametrization of its middle surface. The question we address here is to establish the limit model obtained when $\varepsilon$ goes to zero.

The paper is organized as follows. In Section 2 we introduce the elements of differential geometry necessary to describe the geometry of the shell, we recall the two-dimensional constitutive law of a piezoelectric material (Eq. (4)) and the elastic-electric equilibrium equations of periodically perforated structures (Eq. (8)). In Section 3 we recall the definition and main properties of the unfolding operator $\mathcal{T}^{\varepsilon}$ associated to a reference cell. Finally, in Section 4, we use the operator $\mathcal{T}^{\varepsilon}$ as a tool to establish the strong convergence of the three covariant components of the displacement field and of the electric potential (Eq. (19)) and therefore to obtain the limit model (Eq. (34)); moreover a strong convergence is established for the correctors (Eq. (45)). For the sake of clarity the delicate proof of Korn's inequality for shells in perforated domain is postponed to the Appendix.

\section{TWO-DIMENSIONAL MODEL OF SHELL}

\subsection{Reference configuration}

We denote ${ }^{1}$ by $x_{\alpha}$ the coordinates of a point $x \in \mathbb{R}^{2}$, and by $\partial_{\alpha}:=\partial / \partial x_{\alpha}$ the derivative with respect to $x_{\alpha}$. Let $\Omega \subset \mathbb{R}^{2}$ be a smooth, connex domain with $C^{2}$ boundary. The shell with middle surface $\mathcal{S}$ is defined with the aid of a one-to-one mapping $\theta \in C^{3}(\bar{\Omega})$ with $\mathcal{S}=\theta(\bar{\Omega})$. We assume that for all $x=\left(x_{1}, x_{2}\right) \in \bar{\Omega}$, the two vectors $\boldsymbol{a}_{\alpha}(x):=\partial_{\alpha} \theta(x)$ are linearly independent. Hence, the vectors $\boldsymbol{a}_{\alpha}(x)$ span the tangent plane to the surface at the point $\theta(x) \in \mathcal{S}, x \in \Omega$. Let $\boldsymbol{a}_{3}(x)$ be the unit normal vector to $\mathcal{S}$ at $\theta(x)$ defined by

$$
\boldsymbol{a}_{3}(x)=\frac{\boldsymbol{a}_{1}(x) \times \boldsymbol{a}_{2}(x)}{\left|\boldsymbol{a}_{1}(x) \times \boldsymbol{a}_{2}(x)\right|} .
$$

The three vectors $\boldsymbol{a}_{i}(x)$ form the covariant basis at $\theta(x)$ while the three vectors $\boldsymbol{a}^{i}(x)$ given by relations

$$
\boldsymbol{a}^{i}(x) \cdot \boldsymbol{a}_{j}(x)=\delta_{j}^{i},
$$

form the two dimensional contravariant basis at $\theta(x)$. Let us remark that the vectors $\boldsymbol{a}^{\alpha}(x)$ defined above also span the tangent plane to $\mathcal{S}$ at $\theta(x)$ and that $\boldsymbol{a}_{3}(x)=\boldsymbol{a}_{3}(x)$. Let $a_{\alpha \beta}=\boldsymbol{a}_{\alpha} \cdot \boldsymbol{a}_{\beta}$ and $a^{\alpha \beta}=\boldsymbol{a}^{\alpha} \cdot \boldsymbol{a}^{\beta}$ be the covariant and the contravariant components of the metric tensor of $\mathcal{S}$. The area element along the surface $\mathcal{S}$ is $\sqrt{a} \mathrm{~d} x_{1} \mathrm{~d} x_{2}$ where $a=\operatorname{det}\left(a_{\alpha \beta}\right)$.

\footnotetext{
${ }^{1}$ Throughout this paper, Latin indices and exponents take their values in the set $\{1,2,3\}$, Greek indices and exponents (except $\varepsilon$ ) take their values in the set $\{1,2\}$ and the summation convention with respect to repeated indices and exponents is used. Boldface letters represent vector-valued functions or spaces.
} 
The shell with thickness $2 t$ and middle surface $\mathcal{S}$ is defined by the map

$$
\begin{gathered}
\Theta: \Omega^{t}:=\Omega \times[-t, t] \rightarrow \mathbb{R}^{3}, \\
\Theta\left(x_{1}, x_{2}, x_{3}\right)=\theta\left(x_{1}, x_{2}\right)+x_{3} \boldsymbol{a}_{3}\left(x_{1}, x_{2}\right), \quad \text { for all }\left(x_{1}, x_{2}, x_{3}\right) \in \Omega^{t} .
\end{gathered}
$$

For $t>0$ small enough, the mapping $\Theta$ is injective and the three vectors $\boldsymbol{g}_{i}\left(x_{1}, x_{2}, x_{3}\right)=\partial_{i} \Theta\left(x_{1}, x_{2}, x_{3}\right)$ are linearly independent at each point $\left(x_{1}, x_{2}, x_{3}\right) \in \Omega^{t}$. The vectors $\boldsymbol{g}_{i}\left(x_{1}, x_{2}, x_{3}\right)$ form the three dimensional covariant basis at the point $\Theta\left(x_{1}, x_{2}, x_{3}\right)$. In this way we have defined a shell having the middle surface $\mathcal{S}$ and constant thickness $2 t>0$, i.e., a body whose reference configuration is the set $\overline{\Theta\left(\Omega^{t}\right)}$.

\subsection{Three-dimensional piezoelectricity}

Under the action of applied volume loading $\boldsymbol{f} \in \boldsymbol{L}^{2}\left(\Omega^{t}\right)$ and without electric charges, the shell undergoes an elastic displacement field $\boldsymbol{u}$ and a scalar electric potential $\varphi$ given by the equations below:

$$
\left\{\begin{aligned}
-\operatorname{div} \boldsymbol{\sigma}(\boldsymbol{u}, \varphi) & =\boldsymbol{f} & & \text { in } \Omega^{t}, \\
-\operatorname{div} \boldsymbol{D}(\boldsymbol{u}, \varphi) & =0 & & \text { in } \Omega^{t}
\end{aligned}\right.
$$

where $\boldsymbol{\sigma}=\left(\sigma^{i j}\right)$ is the linearized stress tensor and $\boldsymbol{D}=\left(D^{i}\right)$ is the electric displacements vector defined as

$$
\left\{\begin{aligned}
\sigma^{i j}(\boldsymbol{u}, \varphi) & =c^{i j k l} s_{k l}(\boldsymbol{u})+e^{k i j} \partial_{k} \varphi \\
D^{i}(\boldsymbol{u}, \varphi) & =-e^{i k l} s_{k l}(\boldsymbol{u})+d^{i j} \partial_{j} \varphi
\end{aligned}\right.
$$

and

$$
\left\{\begin{aligned}
(\operatorname{div} \boldsymbol{\sigma}(B u, \varphi))^{j} & =\partial_{i} \sigma^{i j}(\boldsymbol{u}, \varphi), \\
\operatorname{div} \boldsymbol{D}(\boldsymbol{u}, \varphi) & =\partial_{i} D^{i}(\boldsymbol{u}, \varphi),
\end{aligned}\right.
$$

here $\left(s_{i j}\right)$ represents the linearized deformation tensor which is given by

$$
s_{i j}(\boldsymbol{u})=\frac{1}{2}\left(\partial_{i} u_{j}+\partial_{j} u_{i}\right)
$$

Hence, the characteristics of the three dimensional piezoelectric material are given by the elastic tensor $\left(c^{i j k l}\right)$, the dielectric tensor $\left(d^{i j}\right)$ and the piezoelectric coupling tensor $\left(e^{i j k}\right)$. These three tensors have the following properties:

- the elastic tensor $\left(c^{i j k l}\right)$ is symmetric and positive definite, that is,

$$
c^{i j k l}=c^{j i k l}=c^{k l i j}
$$

and there exists $\alpha_{c}>0$ such that $c^{i j k l} X_{i j} X_{k l} \geq \alpha_{c} X_{i j} X_{i j}, \forall X_{i j}=X_{j i} \in \mathbb{R}$;

- the dielectric tensor $\left(d^{i j}\right)$ is symmetric and positive definite, that is, $d^{i j}=d^{j i}$, and there exists $\alpha_{d}>0$ such that $d^{i j} X_{i} X_{j} \geq \alpha_{d} X_{i} X_{i}, \forall X=\left(X_{i}\right) \in \mathbb{R}^{3}$;

- the piezoelectric tensor $\left(e^{i j l}\right)$ is symmetric in the sense that $e^{i j k}=e^{j i k}$.

We assume that the components of the the above three tensors belong to $L^{\infty}\left(\Omega^{t}\right)$.

The variational formulation of the problem (1) reads: find $\boldsymbol{u} \in H_{0}^{1}\left(\Omega^{t} ; \mathbb{R}^{3}\right)$ and $\varphi \in H_{0}^{1}\left(\Omega^{t}\right)$ such that

$$
\left\{\begin{aligned}
\int_{\Omega^{t}}(c(\boldsymbol{u}, \boldsymbol{v})+e(\boldsymbol{v}, \varphi)) \mathrm{d} x & =\int_{\Omega^{t}} \boldsymbol{f} \cdot \boldsymbol{v} \mathrm{d} x & & \forall \boldsymbol{v} \in H_{0}^{1}\left(\Omega^{t} ; \mathbb{R}^{3}\right), \\
\int_{\Omega^{t}}(-e(\boldsymbol{u}, \psi)+d(\varphi, \psi)) \mathrm{d} x & =0 & & \forall \psi \in H_{0}^{1}\left(\Omega^{t}\right),
\end{aligned}\right.
$$


where $c, e$ and $d$ are given by

$$
\left\{\begin{array}{l}
c(\boldsymbol{u}, \boldsymbol{v})=c^{i j k l} s_{i j}(\boldsymbol{u}) s_{k l}(\boldsymbol{v}) \\
e(\boldsymbol{v}, \psi)=e^{i j k} s_{i j}(\boldsymbol{v}) \partial_{k} \psi \\
d(\varphi, \psi)=d^{i j} \partial_{i} \varphi \partial_{j} \psi
\end{array}\right.
$$

\subsection{From three-dimensional equations to two-dimensional equations: the piezoelectric shell equations}

In the case of two-dimensional model of piezoelectric shell, four new tensors are needed. Their expressions in terms of the previous three dimensional ones are given in Haenel [13], equations 3.2.23, 3.2.27:

- the membrane elastic tensor $\left(c_{M}^{\alpha \beta \sigma \tau}\right)$, which is symmetric and positive definite;

- the flexural elastic tensor $\left(c_{F}^{\alpha \beta \sigma \tau}\right)$, which is symmetric and positive definite;

- the dielectric tensor $\left(d^{\alpha \beta}\right)$, which is symmetric and positive definite;

- the piezoelectric tensor $\left(e^{\alpha \beta \sigma}\right)$, which is symmetric in the sense that $e^{\alpha \beta \sigma}=e^{\beta \alpha \sigma}$.

With previous assumptions, we deduce that the components of the four above tensors belong to $L^{\infty}(\Omega)$.

We consider a shell in curvilinear coordinates inspired by a Koiter model for the elastic shells (see [15]) in which the relation between the membrane constraints $T^{\alpha \beta}$, the bending constraints $M^{\alpha \beta}$ and the electric displacements $D^{\sigma}$ are expressed in terms of the three covariant components $\boldsymbol{u}=\left(u_{i}\right)$ of the elastic displacement $\boldsymbol{a}^{i} u_{i}$ and of the electric potential $\varphi$ as follows:

$$
\left\{\begin{aligned}
T^{\alpha \beta}(\boldsymbol{u}, \varphi) & =c_{M}^{\alpha \beta \sigma \tau} \gamma_{\sigma \tau}(\boldsymbol{u})+e^{\alpha \beta \sigma} \partial_{\sigma} \varphi \\
M^{\alpha \beta}(\boldsymbol{u}, \varphi) & =-c_{F}^{\alpha \beta \sigma \tau} \rho_{\sigma \tau}(\boldsymbol{u}) \\
D^{\sigma}(\boldsymbol{u}, \varphi) & =-e^{\alpha \beta \sigma} \gamma_{\alpha \beta}(\boldsymbol{u})+d^{\sigma \tau} \partial_{\tau} \varphi
\end{aligned}\right.
$$

In relations (4), the tensor $\gamma=\left(\gamma_{\alpha \beta}\right)$ represents the two-dimensional linearized change of metric tensor. Its components are given by

$$
\gamma_{\alpha \beta}(\boldsymbol{v})=\frac{1}{2}\left(\partial_{\alpha} v_{\beta}+\partial_{\beta} v_{\alpha}\right)-\Gamma_{\alpha \beta}^{\sigma} v_{\sigma}-b_{\alpha \beta} v_{3}
$$

Also in relations (4), $\rho=\left(\rho_{\alpha \beta}\right)$ denotes the two-dimensional linearized change of curvature tensor. Its components are given by

$$
\rho_{\alpha \beta}(\boldsymbol{v})=\partial_{\alpha \beta}^{2} v_{3}-\Gamma_{\alpha \beta}^{\sigma} \partial_{\sigma} v_{3}-b_{\alpha}^{\sigma} b_{\sigma \beta} v_{3}+b_{\alpha}^{\sigma} \partial_{\beta} v_{\sigma}+b_{\beta}^{\sigma} \partial_{\alpha} v_{\sigma}+\left(\partial_{\beta} b_{\alpha}^{\sigma}-b_{\alpha}^{\tau} \Gamma_{\beta \tau}^{\sigma}-b_{\beta}^{\tau} \Gamma_{\alpha \tau}^{\sigma}\right) v_{\sigma},
$$

where $b_{\alpha \beta}, b_{\alpha}^{\beta}$ represent the covariant and the contravariant components of the curvature tensor of the surface $\mathcal{S}$, they are given by

$$
b_{\alpha \beta}=\boldsymbol{a}_{3} \cdot \partial_{\alpha} \boldsymbol{a}_{\beta}, \quad b_{\alpha}^{\beta}=\boldsymbol{a} 3 \cdot \partial_{\alpha} \boldsymbol{a}^{\beta} .
$$

In (5)-(6), $\left(\Gamma_{\alpha \beta}^{\sigma}\right)$ denote the two-dimensional Christoffel symbols for the surface $\mathcal{S}$, that is, $\Gamma_{\alpha \beta}^{\sigma}=\boldsymbol{a}^{\sigma} \cdot \partial_{\alpha} \boldsymbol{a}_{\beta}$.

On the boundary $\partial \Omega$ we assume that the elastic dispacement field $\boldsymbol{u}$ and the electric potential $\varphi$ verify an homogeneous Dirichlet condition, $\boldsymbol{u}=0$ and $\varphi=0$ on $\partial \Omega$.

Assume that the resultant of the applied mechanics forces $\boldsymbol{p}$ expressed in terms of the mean value of $\boldsymbol{f}$ in the covariant basis satisfies the regularity condition $\boldsymbol{p} \in L^{2}(\Omega)$ and set $\boldsymbol{V}(\Omega)=H_{0}^{1}(\Omega) \times H_{0}^{1}(\Omega) \times H_{0}^{2}(\Omega)$. Then, the couple $(\boldsymbol{u}, \varphi) \in \boldsymbol{V}(\Omega) \times H_{0}^{1}(\Omega)$ is the unique solution of the variational problem (see Haenel [13]):

$$
\left\{\begin{aligned}
\int_{\Omega}\left(c_{K}(\boldsymbol{u}, \boldsymbol{v})+e_{K}(\boldsymbol{v}, \varphi)\right) \sqrt{a} \mathrm{~d} x & =\int_{\Omega} \boldsymbol{p} \cdot \boldsymbol{v} \sqrt{a} \mathrm{~d} x & & \forall \boldsymbol{v} \in \boldsymbol{V}(\Omega), \\
\int_{\Omega}\left(-e_{K}(\boldsymbol{u}, \psi)+d_{K}(\varphi, \psi)\right) \sqrt{a} \mathrm{~d} x & =0 & & \forall \psi \in H_{0}^{1}(\Omega),
\end{aligned}\right.
$$




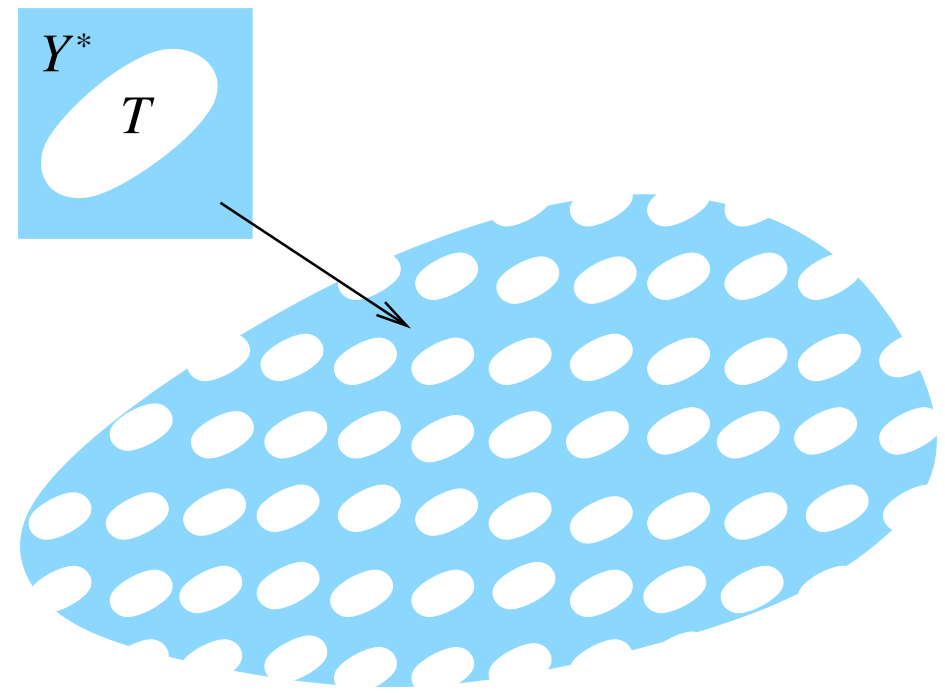

FiguRE 1 . The reference cell $Y^{*}$ and the periodically perforated domain $\Omega^{\varepsilon}$.

where $c_{K}, e_{K}$ and $d_{K}$ are given by

$$
\left\{\begin{aligned}
c_{K}(\boldsymbol{u}, \boldsymbol{v}) & =c_{M}^{\alpha \beta \sigma \tau} \gamma_{\alpha \beta}(\boldsymbol{u}) \gamma_{\sigma \tau}(\boldsymbol{v})+\frac{t 2}{3} c_{F}^{\alpha \beta \sigma \tau} \rho_{\alpha \beta}(\boldsymbol{u}) \rho_{\sigma \tau}(\boldsymbol{v}), \\
e_{K}(\boldsymbol{v}, \psi) & =e^{\alpha \beta \sigma} \gamma_{\alpha \beta}(\boldsymbol{v}) \partial_{\sigma} \psi \\
d_{K}(\varphi, \psi) & =d^{\alpha \beta} \partial_{\alpha} \varphi \partial_{\beta} \psi .
\end{aligned}\right.
$$

\subsection{Geometry of the perforated domain and equilibrium equations}

Let $Y=\left[0,1\left[^{2}\right.\right.$ be the reference cell and let $T$ be the reference hole included in $Y$, that is, $\bar{T} \subset Y$ and $Y^{*}=Y \backslash \bar{T}$ is the part of $Y$ which is occupied by the material. Let $\varepsilon$ be a positive parameter that represents the size of the elementary microstructure that contains the holes. Consider

$$
\mathcal{K}_{\varepsilon}=\left\{k \in \mathbb{Z}^{2} ; \varepsilon(Y+k) \cap \Omega \neq \emptyset\right\}
$$

and set

$$
\widetilde{\Omega}^{\varepsilon}=\bigcup_{k \in \mathcal{K}_{\varepsilon}} \varepsilon\left(Y^{*}+k\right) .
$$

The periodically perforated domain $\Omega^{\varepsilon}$ (see Fig. 1) is defined as

$$
\Omega^{\varepsilon}=\Omega \cap \widetilde{\Omega}^{\varepsilon} .
$$

Remark that $\widetilde{\Omega}^{\varepsilon}$ is the smallest union of $\varepsilon Y^{*}$ cells that contains $\Omega^{\varepsilon}$.

We are now in position to formulate our problem. Let us first introduce the functional spaces

$$
\begin{aligned}
V\left(\Omega^{\varepsilon}\right) & =\left\{\phi \in H^{1}\left(\Omega^{\varepsilon}\right) ; \phi=0 \text { on } \partial \Omega \cap \bar{\Omega}^{\varepsilon}\right\}, \\
W\left(\Omega^{\varepsilon}\right) & =\left\{\phi \in H^{2}\left(\Omega^{\varepsilon}\right) ; \phi=0 \text { on } \partial \Omega \cap \bar{\Omega}^{\varepsilon}\right\}, \\
V\left(\Omega^{\varepsilon}\right) & =V\left(\Omega^{\varepsilon}\right) \times V\left(\Omega^{\varepsilon}\right) \times W\left(\Omega^{\varepsilon}\right) .
\end{aligned}
$$

Since the reference configuration $\Omega^{\varepsilon}$ of the structure and the characteristic tensors $c_{K}^{\varepsilon}, d_{K}^{\varepsilon}, e_{K}^{\varepsilon}$ depend upon $\varepsilon$, the solution $\left(\boldsymbol{u}^{\varepsilon}, \varphi^{\varepsilon}\right)$ also depends upon $\varepsilon$. The equilibrium equations satisfied by the couple $\left(\boldsymbol{u}^{\varepsilon}, \varphi^{\varepsilon}\right) \in \boldsymbol{V}\left(\Omega^{\varepsilon}\right) \times$ 
$V\left(\Omega^{\varepsilon}\right)$ are given by

$$
\left\{\begin{aligned}
\int_{\Omega^{\varepsilon}}\left(c_{K}^{\varepsilon}\left(\boldsymbol{u}^{\varepsilon}, \boldsymbol{v}\right)+e_{K}^{\varepsilon}\left(\boldsymbol{v}, \varphi^{\varepsilon}\right)\right) \sqrt{a} \mathrm{~d} x=\int_{\Omega^{\varepsilon}} \boldsymbol{p} \cdot \boldsymbol{v} \sqrt{a} \mathrm{~d} x & & \forall \boldsymbol{v} \in \boldsymbol{V}\left(\Omega^{\varepsilon}\right), \\
\int_{\Omega^{\varepsilon}}\left(-e_{K}^{\varepsilon}\left(\boldsymbol{u}^{\varepsilon}, \psi\right)+d_{K}^{\varepsilon}\left(\varphi^{\varepsilon}, \psi\right)\right) \sqrt{a} \mathrm{~d} x=0 & & \forall \psi \in V\left(\Omega^{\varepsilon}\right),
\end{aligned}\right.
$$

where, by analogy with the previous notations, $c_{K}^{\varepsilon}, e_{K}^{\varepsilon}$ and $d_{K}^{\varepsilon}$ are defined as

$$
\left\{\begin{aligned}
c_{K}^{\varepsilon}(\boldsymbol{u}, \boldsymbol{v}) & =c_{M}^{\varepsilon, \alpha \beta \sigma \tau} \gamma_{\alpha \beta}(\boldsymbol{u}) \gamma_{\sigma \tau}(\boldsymbol{v})+\frac{t 2}{3} c_{F}^{\varepsilon, \alpha \beta \sigma \tau} \rho_{\alpha \beta}(\boldsymbol{u}) \rho_{\sigma \tau}(\boldsymbol{v}) \\
e_{K}^{\varepsilon}(\boldsymbol{v}, \psi) & =e^{\varepsilon, \alpha \beta \sigma} \gamma_{\alpha \beta}(\boldsymbol{v}) \partial_{\sigma} \psi \\
d_{K}^{\varepsilon}(\varphi, \psi) & =d^{\varepsilon, \alpha \beta} \partial_{\alpha} \varphi \partial_{\beta} \psi
\end{aligned}\right.
$$

\section{Periodic unfolding operator}

Our aim in this paper is to study the limit of the couple $\left(\boldsymbol{u}^{\varepsilon}, \varphi^{\varepsilon}\right)$ as $\varepsilon \rightarrow 0$. For this purpose, we use the periodic unfolding method introduced by Cioranescu et al. [7]. The main feature here consists in the presence of the periodic perforations that determine a more complicated limit constitutive law. The interest is also due to the fact that the two-dimensional linearized change of metric and curvature tensors present different order of derivatives of their arguments. We will show that this gives rise to local problems of different nature than the global one.

For $x \in \mathbb{R}^{2}$ we denote by $[x]$ the unique integer combination such that $x-[x] \in Y$ and set $\{x\}=x-[x] \in Y$. In this manner, for any $x \in \mathbb{R}^{2}$ and $\varepsilon>0$, we have:

$$
x=\varepsilon\left(\left[\frac{x}{\varepsilon}\right]+\left\{\frac{x}{\varepsilon}\right\}\right) .
$$

Definition 3.1. The unfolding operator $\mathcal{T}^{\varepsilon}: L^{1}\left(\Omega^{\varepsilon}\right) \rightarrow L^{1}\left(\Omega \times Y^{*}\right)$ is given by

$$
\mathcal{T}^{\varepsilon}(v)(x, y)=v\left(\varepsilon\left[\frac{x}{\varepsilon}\right]+\varepsilon y\right), \text { for all } v \in L^{1}\left(\Omega^{\varepsilon}\right) \text { extended by zero in } \widetilde{\Omega}^{\varepsilon} \backslash \Omega^{\varepsilon},
$$

where $x$ is the so-called "slow variable" and $y$ is the "rapid variable".

Obviously, $\mathcal{T}^{\varepsilon}$ is a linear operator and

$$
\mathcal{T}^{\varepsilon}(v w)=\mathcal{T}^{\varepsilon}(v) \mathcal{T}^{\varepsilon}(w), \quad \text { for all } v, w \in L^{1}\left(\Omega^{\varepsilon}\right) .
$$

From the definition of $\widetilde{\Omega}^{\varepsilon}$ we get the exact integration formula

$$
\int_{\Omega} v \mathrm{~d} x=\int_{\widetilde{\Omega}^{\varepsilon} \times Y^{*}} \mathcal{T}^{\varepsilon}(v) \mathrm{d} x \mathrm{~d} y, \quad \text { for all } v \in L^{1}\left(\Omega^{\varepsilon}\right) .
$$

Let $\mathcal{O}^{\varepsilon}$ be the set of all $\varepsilon Y^{*}$ cells that intersect the boundary $\partial \Omega$, that is,

$$
\mathcal{O}^{\varepsilon}=\left\{x \in \widetilde{\Omega}^{\varepsilon} ;\left(\varepsilon\left[\frac{x}{\varepsilon}\right]+\varepsilon Y\right) \cap \partial \Omega \neq \emptyset\right\} .
$$

Then, for all $v \in L^{1}\left(\Omega^{\varepsilon}\right)$ we have

$$
\left|\int_{\Omega^{\varepsilon}} v \mathrm{~d} x-\int_{\Omega \times Y^{*}} \mathcal{T}^{\varepsilon}(v) \mathrm{d} x \mathrm{~d} y\right| \leq \int_{\mathcal{O}^{\varepsilon}}|v| \mathrm{d} x .
$$


For a function $u=u(x, y)$ we denote by $\nabla_{x} u$ and $\nabla_{y} u$ the gradient of $u$ with respect to $x$ and $y$ variable respectively and $\partial_{i, y} u$ represents the derivative of $u$ with respect to $y_{i}$. For $k=1,2$ we denote by $H_{\mathrm{per}}^{k}\left(Y^{*}\right)$ the set of all functions of $H^{k}\left(Y^{*}\right)$ with vanishing mean value, extended by $Y$-periodicity.

The main properties of the unfolding operator $\mathcal{T}^{\varepsilon}$ are described below.

Proposition 3.2. The following properties hold true:

(i) For any $v \in L^{1}\left(\Omega^{\varepsilon}\right), v \geq 0$ we have

$$
\int_{\Omega \times Y^{*}} \mathcal{T}^{\varepsilon}(v) \mathrm{d} x \mathrm{~d} y \leq \int_{\Omega^{\varepsilon}} v \mathrm{~d} x .
$$

(ii) If $v \in L^{2}(\Omega)$ then $\mathcal{T}^{\varepsilon}(v) \rightarrow v$ strongly in $L^{2}\left(\Omega \times Y^{*}\right)$.

(iii) Let $\left\{v^{\varepsilon}\right\}_{\varepsilon}$ be a uniformly bounded sequence in $L^{p}\left(\Omega^{\varepsilon}\right), p>1$, that is, $\left\|v^{\varepsilon}\right\|_{L^{p}\left(\Omega^{\varepsilon}\right)} \leq C$ with $C>0$ independent on $\varepsilon$. Then

$$
\lim _{\varepsilon \rightarrow 0} \int_{\mathcal{O}^{\varepsilon}}\left|v^{\varepsilon}\right| \mathrm{d} x=0 .
$$

(iv) Let $\left\{v^{\varepsilon}\right\}_{\varepsilon}$ be a uniformly bounded sequence in $L^{2}\left(\Omega^{\varepsilon}\right)$. Then, there exists $v \in L^{2}\left(\Omega \times Y^{*}\right)$ such that, up to a subsequence, we have

$$
\mathcal{T}^{\varepsilon}\left(v^{\varepsilon}\right) \rightarrow v \text { weakly in } L^{2}\left(\Omega \times Y^{*}\right) \text {. }
$$

(v) Let $\left\{v^{\varepsilon}\right\}_{\varepsilon}$ be a uniformly bounded sequence in $H^{1}\left(\Omega^{\varepsilon}\right)$. Then, there exist $v \in H^{1}(\Omega)$ and a corrector field $\bar{v} \in L^{2}\left(\Omega, H_{\mathrm{per}}^{1}\left(Y^{*}\right)\right)$, such that, up to a subsequence, the following convergences hold

$$
\left\{\begin{array}{clrl}
\mathcal{T}^{\varepsilon}\left(v^{\varepsilon}\right) & \rightarrow v & & \text { strongly in } L^{2}\left(\Omega \times Y^{*}\right), \\
\mathcal{T}^{\varepsilon}\left(\nabla_{x} v^{\varepsilon}\right) & \rightarrow \nabla_{x} v+\nabla_{y} \bar{v} & & \text { weakly in } L^{2}\left(\Omega \times Y^{*} ; \mathbb{R}^{2}\right) .
\end{array}\right.
$$

(vi) Let $\left\{v^{\varepsilon}\right\}_{\varepsilon}$ be a uniformly bounded sequence in $H^{2}\left(\Omega^{\varepsilon}\right)$. Then, there exist $v \in H^{2}(\Omega)$ and a corrector field $\bar{v} \in L^{2}\left(\Omega, H_{\mathrm{per}}^{2}\left(Y^{*}\right)\right)$ such that, up to a subsequence, the following convergences hold

$$
\left\{\begin{aligned}
\mathcal{T}^{\varepsilon}\left(v^{\varepsilon}\right) & \rightarrow v & & \text { strongly in } L^{2}\left(\Omega \times Y^{*}\right), \\
\mathcal{T}^{\varepsilon}\left(\nabla_{x} v^{\varepsilon}\right) & \rightarrow \nabla_{x} v & & \text { strongly in } L^{2}\left(\Omega \times Y^{*} ; \mathbb{R}^{2}\right), \\
\mathcal{T}^{\varepsilon}\left(\nabla_{x}^{2} v^{\varepsilon}\right) & \rightarrow \nabla_{x}^{2} v+\nabla_{y}^{2} \bar{v} & & \text { weakly in } L^{2}\left(\Omega \times Y^{*} ; \mathbb{R}^{2} \times \mathbb{R}^{2}\right) .
\end{aligned}\right.
$$

Proof. The statement $(i)$ is a direct consequence of $(10)$ while $(i i)-(i v)$ are proved in $[7,9]$ and $(v)$ in $[7,11]$. We present here only the proof of $(v i)$. Let $\left\{v^{\varepsilon}\right\}_{\varepsilon}$ be an uniformly bounded sequence in $H^{2}\left(\Omega^{\varepsilon}\right)$. Since $\left\{v^{\varepsilon}\right\}$ is uniformly bounded in $H^{1}\left(\Omega^{\varepsilon}\right)$, by $(i v)$ there exist $v \in H^{1}(\Omega)$ and $\widehat{v} \in L 2\left(\Omega, H_{\mathrm{per}}^{1}\left(Y^{*}\right)\right)$ such that

$$
\mathcal{T}^{\varepsilon}\left(v^{\varepsilon}\right) \rightarrow v \quad \text { strongly in } L^{2}\left(\Omega \times Y^{*}\right),
$$

and

$$
\mathcal{T}^{\varepsilon}\left(\nabla_{x} v^{\varepsilon}\right) \rightarrow \nabla_{x} v+\nabla_{y} \widehat{v} \quad \text { weakly in } L^{2}\left(\Omega \times Y^{*} ; \mathbb{R}^{2}\right) .
$$

On the other hand, $\left\{\partial_{i} v^{\varepsilon}\right\}$ is uniformly bounded in $H^{1}\left(\Omega^{\varepsilon}\right)$. Hence, by $(i v)$ there exist $w_{i} \in H^{1}(\Omega)$ and $\widehat{w}_{i} \in L^{2}\left(\Omega \times Y^{*}\right)$ such that

$$
\mathcal{T}^{\varepsilon}\left(\partial_{i} v^{\varepsilon}\right) \rightarrow w_{i} \quad \text { strongly in } L^{2}\left(\Omega \times Y^{*}\right),
$$

and

$$
\mathcal{T}^{\varepsilon}\left(\nabla_{x}\left(\partial_{i} v^{\varepsilon}\right)\right) \rightarrow \nabla_{x} w_{i}+\nabla_{y} \widehat{w}_{i} \quad \text { weakly in } L^{2}\left(\Omega \times Y^{*} ; \mathbb{R}^{2}\right) .
$$


From (13) and (14) we deduce $\partial_{i} v+\partial_{i, y} \widehat{v}=w_{i}$ in $\Omega \times Y^{*}$. Since $\partial_{i} v$ and $w_{i}$ depend only on $x$, we obtain $\nabla_{y} \widehat{v}=0$. This means $\partial_{i} v=w_{i}$ in $\Omega$, that is, $v \in H^{2}(\Omega)$.

By (15) we obtain

$$
\mathcal{T}^{\varepsilon}\left(\partial_{i j}^{2} v^{\varepsilon}\right) \rightarrow \partial_{i j}^{2} v+\partial_{j, y} \widehat{w}_{i} \quad \text { weakly in } L^{2}\left(\Omega \times Y^{*}\right) .
$$

Interchanging $i$ and $j$ in (16) we also have

$$
\mathcal{T}^{\varepsilon}\left(\partial_{j i}^{2} v^{\varepsilon}\right) \rightarrow \partial_{j i}^{2} v+\partial_{i, y} \widehat{w}_{j} \quad \text { weakly in } L^{2}\left(\Omega \times Y^{*}\right) .
$$

Since $v \in H^{2}(\Omega)$, from (16) and (17) one obtains

$$
\partial_{j, y} \widehat{w}_{i}=\partial_{i, y} \widehat{w}_{j} \quad \text { in } \Omega \times Y^{*} .
$$

A classical result states that if $\widehat{\boldsymbol{w}}=\left(\widehat{w}_{i}\right)$ satisfies (18) in a simply connected domain, then $\widehat{\boldsymbol{w}}$ is a gradient of a vector field. This result also holds for perforated domains provided that the set of perforations has a Lipschitz boundary. Therefore, there exists $\bar{v} \in L^{2}\left(\Omega ; H_{\mathrm{per}}^{2}\left(Y^{*}\right)\right)$ such that $\widehat{\boldsymbol{w}}=\nabla_{y} \bar{v}$. Now (16) yields

$$
\mathcal{T}^{\varepsilon}\left(\nabla_{x}^{2} v^{\varepsilon}\right) \rightarrow \nabla_{x}^{2} v+\nabla_{y}^{2} \bar{v} \quad \text { weakly in } L^{2}\left(\Omega \times Y^{*} ; \mathbb{R}^{2} \times \mathbb{R}^{2}\right) .
$$

This completes the proof.

\section{Homogenization of PIEZOelectric SHELL}

We assume that there exist four tensors $c_{M}, c_{F}, d, e$ which depend only on the microscopic variable $y$, such that

$$
\mathcal{T}^{\varepsilon}\left(c_{M}^{\varepsilon, \alpha \beta \lambda \mu}\right)(x, y)=c_{M}^{\alpha \beta \lambda \mu}(y), \quad \text { for all }(x, y) \in \Omega \times Y^{*},
$$

and similarly for all tensors. Let $\boldsymbol{V}_{\text {per }}\left(Y^{*}\right)=H_{\text {per }}^{1}\left(Y^{*}\right) \times H_{\text {per }}^{1}\left(Y^{*}\right) \times H_{\text {per }}^{2}\left(Y^{*}\right)$.

We first prove the following result.

Theorem 4.1. Let $\left(\boldsymbol{u}^{\varepsilon}, \varphi^{\varepsilon}\right) \in \boldsymbol{V}\left(\Omega^{\varepsilon}\right) \times V\left(\Omega^{\varepsilon}\right)$ be the unique solution of (8). Then, there exist $(\boldsymbol{u}, \varphi) \in$ $\boldsymbol{V}(\Omega) \times H_{0}^{1}(\Omega)$ and two corrector fields $\overline{\boldsymbol{u}} \in \boldsymbol{L}^{2}\left(\Omega, \boldsymbol{V}_{\mathrm{per}}\left(Y^{*}\right)\right), \bar{\varphi} \in L^{2}\left(\Omega, H_{\mathrm{per}}^{1}\left(Y^{*}\right)\right)$ defined by the following convergence

$$
\left\{\begin{array}{clrl}
\mathcal{T}^{\varepsilon}\left(\boldsymbol{u}^{\varepsilon}\right) & \rightarrow \boldsymbol{u} & \text { strongly in } \boldsymbol{L}^{2}\left(\Omega \times Y^{*}\right) \\
\mathcal{T}^{\varepsilon}\left(\varphi^{\varepsilon}\right) \rightarrow \varphi & \text { strongly in } L^{2}\left(\Omega \times Y^{*}\right) \\
\mathcal{T}^{\varepsilon}\left(\gamma_{\alpha \beta}\left(\boldsymbol{u}^{\varepsilon}\right)\right) & -\gamma_{\alpha \beta}(\boldsymbol{u})+s_{\alpha \beta, y}(\overline{\boldsymbol{u}}) & & \text { weakly in } L^{2}\left(\Omega \times Y^{*}\right) \\
\mathcal{T}^{\varepsilon}\left(\rho_{\alpha \beta}\left(\boldsymbol{u}^{\varepsilon}\right)\right) & -\rho_{\alpha \beta}(\boldsymbol{u})+r_{\alpha \beta, y}(\overline{\boldsymbol{u}}) & & \text { weakly in } L^{2}\left(\Omega \times Y^{*}\right) \\
\mathcal{T}^{\varepsilon}\left(\nabla_{x} \varphi^{\varepsilon}\right) & -\nabla_{x} \varphi+\nabla_{y} \bar{\varphi} & \text { weakly in } L^{2}\left(\Omega \times Y^{*} ; \mathbb{R}^{2}\right)
\end{array}\right.
$$

with $s_{\alpha \beta, y}(\overline{\boldsymbol{u}})=\frac{1}{2}\left(\partial_{\alpha, y} \bar{u}_{\beta}+\partial_{\beta, y} \bar{u}_{\alpha}\right)$ and $r_{\alpha \beta, y}(\overline{\boldsymbol{u}})=\partial_{\alpha \beta, y}^{2} \bar{u}_{3}+b_{\alpha}^{\sigma} \partial_{\beta, y} \bar{u}_{\sigma}+b_{\beta}^{\sigma} \partial_{\alpha, y} \bar{u}_{\sigma}$.

The limit fields $(\boldsymbol{u}, \varphi)$ and $(\overline{\boldsymbol{u}}, \bar{\varphi})$ are the unique solution of the following variational problem posed for all $\boldsymbol{v} \in \boldsymbol{V}(\Omega), \overline{\boldsymbol{v}} \in \boldsymbol{L}^{2}\left(\Omega, \boldsymbol{V}_{\mathrm{per}}\left(Y^{*}\right)\right), \psi \in H_{0}^{1}(\Omega), \bar{\psi} \in L^{2}\left(\Omega, H_{\mathrm{per}}^{1}\left(Y^{*}\right)\right):$

$$
\left\{\begin{array}{l}
\int_{\Omega \times Y^{*}}\left(c_{M}^{\alpha \beta \sigma \tau}\left(\gamma_{\alpha \beta}(\boldsymbol{u})+s_{\alpha \beta, y}(\overline{\boldsymbol{u}})\right)\left(\gamma_{\sigma \tau}(\boldsymbol{v})+s_{\sigma \tau, y}(\overline{\boldsymbol{v}})\right)\right. \\
\left.+\frac{t^{2}}{3} c_{F}^{\alpha \beta \sigma \tau}\left(\rho_{\alpha \beta}(\boldsymbol{u})+r_{\alpha \beta, y}(\overline{\boldsymbol{u}})\right)\left(\rho_{\sigma \tau}(\boldsymbol{v})+r_{\sigma \tau, y}(\overline{\boldsymbol{v}})\right)\right) \sqrt{a} \mathrm{~d} x \mathrm{~d} y \\
+\int_{\Omega \times Y^{*}} e^{\alpha \beta \sigma}\left(\gamma_{\alpha \beta}(\boldsymbol{v})+s_{\alpha \beta, y}(\overline{\boldsymbol{v}})\right)\left(\partial_{\sigma} \varphi+\partial_{\sigma, y} \bar{\varphi}\right) \sqrt{a} \mathrm{~d} x \mathrm{~d} y=\left|Y^{*}\right| \int_{\Omega} \boldsymbol{p} \cdot \boldsymbol{v} \sqrt{a} \mathrm{~d} x, \\
\int_{\Omega \times Y^{*}}\left(-e^{\alpha \beta \sigma}\left(\gamma_{\alpha \beta}(\boldsymbol{u})+s_{\alpha \beta, y}(\overline{\boldsymbol{u}})\right)\left(\partial_{\sigma} \psi+\partial_{\sigma, y} \bar{\psi}\right)+d^{\alpha \beta}\left(\partial_{\alpha} \varphi+\partial_{\alpha, y} \bar{\varphi}\right)\left(\partial_{\beta} \psi+\partial_{\beta, y} \bar{\psi}\right)\right) \sqrt{a} \mathrm{~d} x \mathrm{~d} y=0 .
\end{array}\right.
$$


We shall prove (see Thm. 4.4) that the last three convergences in (19) are actually strong.

Proof. We divide the proof into three steps.

Step 1. A priori estimates. Taking $(\boldsymbol{v}, \psi)=\left(\boldsymbol{u}^{\varepsilon}, \varphi^{\varepsilon}\right)$ in (8) we have

$$
\left\{\begin{aligned}
& \int_{\Omega^{\varepsilon}}\left(c_{K}^{\varepsilon}\left(\boldsymbol{u}^{\varepsilon}, \boldsymbol{u}^{\varepsilon}\right)+e_{K}^{\varepsilon}\left(\boldsymbol{u}^{\varepsilon}, \varphi^{\varepsilon}\right)\right) \sqrt{a} \mathrm{~d} x=\int_{\Omega^{\varepsilon}} \boldsymbol{p} \cdot \boldsymbol{u}^{\varepsilon} \sqrt{a} \mathrm{~d} x \\
& \int_{\Omega^{\varepsilon}}\left(-e_{K}^{\varepsilon}\left(\boldsymbol{u}^{\varepsilon}, \varphi^{\varepsilon}\right)+d_{K}^{\varepsilon}\left(\varphi^{\varepsilon}, \varphi^{\varepsilon}\right)\right) \sqrt{a} \mathrm{~d} x=0 .
\end{aligned}\right.
$$

By summation we obtain

$$
\int_{\Omega^{\varepsilon}}\left(c_{K}^{\varepsilon}\left(\boldsymbol{u}^{\varepsilon}, \boldsymbol{u}^{\varepsilon}\right)+d_{K}^{\varepsilon}\left(\varphi^{\varepsilon}, \varphi^{\varepsilon}\right)\right) \sqrt{a} \mathrm{~d} x=\int_{\Omega^{\varepsilon}} \boldsymbol{p} \cdot \boldsymbol{u}^{\varepsilon} \sqrt{a} \mathrm{~d} x
$$

that is,

$$
\int_{\Omega^{\varepsilon}}\left(c_{M}^{\varepsilon, \alpha \beta \sigma \tau} \gamma_{\alpha \beta}\left(\boldsymbol{u}^{\varepsilon}\right) \gamma_{\sigma \tau}\left(\boldsymbol{u}^{\varepsilon}\right)+\frac{t^{2}}{3} c_{F}^{\varepsilon, \alpha \beta \sigma \tau} \rho_{\alpha \beta}\left(\boldsymbol{u}^{\varepsilon}\right) \rho_{\sigma \tau}\left(\boldsymbol{u}^{\varepsilon}\right)+d^{\varepsilon, \alpha \beta} \partial_{\alpha} \varphi^{\varepsilon} \partial_{\beta} \varphi^{\varepsilon}\right) \sqrt{a} \mathrm{~d} x=\int_{\Omega^{\varepsilon}} \boldsymbol{p} \cdot \boldsymbol{u}^{\varepsilon} \sqrt{a} \mathrm{~d} x .
$$

Using Hölder's inequality and the fact that the membrane, flexural and dielectric tensors are positive definite, we obtain the estimate

$$
\sum_{\alpha, \beta}\left\{\left\|\rho_{\alpha \beta}\left(\boldsymbol{u}^{\varepsilon}\right)\right\|_{L^{2}\left(\Omega^{\varepsilon}\right)}^{2}+\left\|\gamma_{\alpha \beta}\left(\boldsymbol{u}^{\varepsilon}\right)\right\|_{L^{2}\left(\Omega^{\varepsilon}\right)}^{2}\right\}+\sum_{\alpha}\left\|\partial_{\alpha} \varphi^{\varepsilon}\right\|_{L^{2}\left(\Omega^{\varepsilon}\right)}^{2} \leq C_{1}\left\|\boldsymbol{u}^{\varepsilon}\right\|_{L^{2}\left(\Omega^{\varepsilon}\right)}
$$

where $C_{1}>0$ is independent on $\varepsilon$.

On the other hand, by Korn's inequality for shells in the case of perforated domains (see Thm. 6.2 in the Appendix) we have

$$
\left\|u_{1}^{\varepsilon}\right\|_{H^{1}\left(\Omega^{\varepsilon}\right)}^{2}+\left\|u_{2}^{\varepsilon}\right\|_{H^{1}\left(\Omega^{\varepsilon}\right)}^{2}+\left\|u_{3}^{\varepsilon}\right\|_{H^{2}\left(\Omega^{\varepsilon}\right)}^{2} \leq C_{2} \sum_{\alpha, \beta}\left\{\left\|\rho_{\alpha \beta}\left(\boldsymbol{u}^{\varepsilon}\right)\right\|_{L^{2}\left(\Omega^{\varepsilon}\right)}^{2}+\left\|\gamma_{\alpha \beta}\left(\boldsymbol{u}^{\varepsilon}\right)\right\|_{L^{2}\left(\Omega^{\varepsilon}\right)}^{2}\right\}
$$

with $C_{2}$ independent on $\varepsilon$. Combining (22) and (23) and using Poincaré's inequality we get

$$
\left\|u_{1}^{\varepsilon}\right\|_{H^{1}\left(\Omega^{\varepsilon}\right)}+\left\|u_{2}^{\varepsilon}\right\|_{H^{1}\left(\Omega^{\varepsilon}\right)}+\left\|u_{3}^{\varepsilon}\right\|_{H^{2}\left(\Omega^{\varepsilon}\right)}+\left\|\varphi^{\varepsilon}\right\|_{H^{1}\left(\Omega^{\varepsilon}\right)} \leq C,
$$

where $C$ is a positive constant that does not depend on $\varepsilon$.

Step 2. Weak convergence. In view of Proposition $3.2(v)-(v i)$, there exist a piezoelectric field $(\boldsymbol{u}, \varphi) \in$ $\boldsymbol{V}(\Omega) \times H_{0}^{1}(\Omega)$ and two corrector fields $\overline{\boldsymbol{u}} \in \boldsymbol{L}^{2}\left(\Omega, \boldsymbol{V}_{\mathrm{per}}\left(Y^{*}\right)\right), \bar{\varphi} \in L^{2}\left(\Omega, H_{\mathrm{per}}^{1}\left(Y^{*}\right)\right)$ such that

$$
\left\{\begin{aligned}
\mathcal{T}^{\varepsilon}\left(\boldsymbol{u}^{\varepsilon}\right) & \rightarrow \boldsymbol{u} & & \text { strongly in } \boldsymbol{L}^{2}\left(\Omega \times Y^{*}\right), \\
\mathcal{T}^{\varepsilon}\left(\varphi^{\varepsilon}\right) & \rightarrow \varphi & & \text { strongly in } L^{2}\left(\Omega \times Y^{*}\right), \\
\mathcal{T}^{\varepsilon}\left(\nabla_{x} u_{\alpha}^{\varepsilon}\right) & \rightarrow \nabla_{x} u_{\alpha}+\nabla_{y} \bar{u}_{\alpha} & & \text { weakly in } L^{2}\left(\Omega \times Y^{*} ; \mathbb{R}^{2}\right), \\
\mathcal{T}^{\varepsilon}\left(\nabla_{x} u_{3}^{\varepsilon}\right) & \rightarrow \nabla_{x} u_{3} & & \text { strongly in } L^{2}\left(\Omega \times Y^{*} ; \mathbb{R}^{2}\right), \\
\mathcal{T}^{\varepsilon}\left(\nabla_{x}^{2} u_{3}^{\varepsilon}\right) & \rightarrow \nabla_{x}^{2} u_{3}+\nabla_{y}^{2} \bar{u}_{3} & & \text { weakly in } L^{2}\left(\Omega \times Y^{*} ; \mathbb{R}^{2} \times \mathbb{R}^{2}\right) \\
\mathcal{T}^{\varepsilon}\left(\nabla_{x} \varphi^{\varepsilon}\right) & \rightarrow \nabla_{x} \varphi+\nabla_{y} \bar{\varphi} & & \text { weakly in } L^{2}\left(\Omega \times Y^{*} ; \mathbb{R}^{2}\right)
\end{aligned}\right.
$$

From (24) we get

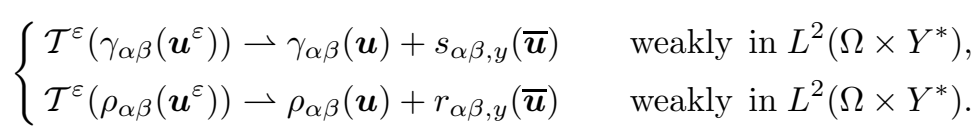


Step 3. Limit coupled problems. Let us first take test functions in (8) of the form $(\boldsymbol{v}, \psi)$ with $\boldsymbol{v}=\left(v_{i}\right)$ and $v_{i}, \psi \in C_{0}^{\infty}(\Omega)$. Then

$$
\left\{\begin{aligned}
\mathcal{T}^{\varepsilon}\left(\gamma_{\alpha \beta}(\boldsymbol{v})\right) & \rightarrow \gamma_{\alpha \beta}(\boldsymbol{v}) & & \text { strongly in } L^{2}\left(\Omega \times Y^{*}\right), \\
\mathcal{T}^{\varepsilon}\left(\rho_{\alpha \beta}(\boldsymbol{v})\right) & \rightarrow \rho_{\alpha \beta}(\boldsymbol{v}) & & \text { strongly in } L^{2}\left(\Omega \times Y^{*}\right), \\
\mathcal{T}^{\varepsilon}\left(\nabla_{x} \psi\right) & \rightarrow \nabla_{x} \psi & & \text { strongly in } L^{2}\left(\Omega \times Y^{*} ; \mathbb{R}^{2}\right) .
\end{aligned}\right.
$$

By (11), Proposition 3.2 ( iii) and (24)-(25) we pass to the limit in order to obtain the following variational problem for all $(\boldsymbol{v}, \psi)$ :

$$
\left\{\begin{array}{l}
\int_{\Omega \times Y^{*}}\left\{c_{M}^{\alpha \beta \sigma \tau}\left(\gamma_{\alpha \beta}(\boldsymbol{u})+s_{\alpha \beta, y}(\overline{\boldsymbol{u}})\right) \gamma_{\sigma \tau}(\boldsymbol{v})+\frac{t^{2}}{3} c_{F}^{\alpha \beta \sigma \tau}\left(\rho_{\alpha \beta}(\boldsymbol{u})+r_{\alpha \beta, y}(\overline{\boldsymbol{u}})\right) \rho_{\sigma \tau}(\boldsymbol{v})\right\} \sqrt{a} \mathrm{~d} x \mathrm{~d} y \\
+\int_{\Omega \times Y^{*}} e^{\alpha \beta \sigma}\left(\partial_{\sigma} \varphi+\partial_{\sigma, y} \bar{\varphi}\right) \gamma_{\alpha \beta}(\boldsymbol{v}) \sqrt{a} \mathrm{~d} x \mathrm{~d} y=\left|Y^{*}\right| \int_{\Omega} \boldsymbol{p} \cdot \boldsymbol{v} \sqrt{a} \mathrm{~d} x, \\
\int_{\Omega \times Y^{*}}\left\{-e^{\alpha \beta \sigma}\left(\gamma_{\alpha \beta}(\boldsymbol{u})+s_{\alpha \beta, y}(\overline{\boldsymbol{u}})\right) \partial_{\sigma} \psi+d^{\alpha \beta}\left(\partial_{\alpha} \varphi+\partial_{\alpha, y} \bar{\varphi}\right) \partial_{\beta} \psi\right\} \sqrt{a} \mathrm{~d} x \mathrm{~d} y=0 .
\end{array}\right.
$$

By density, the above relations hold for all $(\boldsymbol{v}, \psi) \in \boldsymbol{V}(\Omega) \times H_{0}^{1}(\Omega)$.

We now take test functions $\boldsymbol{v}$ and $\psi$ in (8) of the form

$$
\begin{aligned}
& \boldsymbol{v}(x)=\boldsymbol{v}^{\varepsilon}(x)=\left(\varepsilon v_{1}(x) w_{1}\left(\left\{\frac{x}{\varepsilon}\right\}\right), \varepsilon v_{2}(x) w_{2}\left(\left\{\frac{x}{\varepsilon}\right\}\right), \varepsilon^{2} v_{3}(x) w_{3}\left(\left\{\frac{x}{\varepsilon}\right\}\right)\right) \\
& \psi(x)=\psi^{\varepsilon}(x)=\varepsilon \psi(x) \phi\left(\left\{\frac{x}{\varepsilon}\right\}\right)
\end{aligned}
$$

with $v_{i}, \psi \in C_{0}^{\infty}(\Omega)$ and $w_{i}, \phi \in C_{\text {per }}^{\infty}\left(\bar{Y}^{*}\right)$. One can easily check that

$$
\begin{aligned}
\partial_{\alpha} v_{\beta}^{\varepsilon}(x) & =\varepsilon w_{\beta}\left(\left\{\frac{x}{\varepsilon}\right\}\right) \partial_{\alpha} v_{\beta}(x)+v_{\beta}(x) \partial_{\alpha} w_{\beta}\left(\left\{\frac{x}{\varepsilon}\right\}\right) \\
\partial_{\alpha \beta} v_{3}^{\varepsilon}(x) & =\varepsilon^{2} w_{3}\left(\left\{\frac{x}{\varepsilon}\right\}\right) \partial_{\alpha \beta} v_{3}(x)+\varepsilon \partial_{\alpha} v_{3}(x) \partial_{\beta} w_{3}\left(\left\{\frac{x}{\varepsilon}\right\}\right)+\varepsilon \partial_{\beta} v_{3}(x) \partial_{\alpha} w_{3}\left(\left\{\frac{x}{\varepsilon}\right\}\right)+v_{3}(x) \partial_{\alpha \beta} w_{3}\left(\left\{\frac{x}{\varepsilon}\right\}\right) \\
\partial_{\alpha} \psi^{\varepsilon}(x) & =\varepsilon \phi\left(\left\{\frac{x}{\varepsilon}\right\}\right) \partial_{\alpha} \psi(x)+\psi(x) \partial_{\alpha} \phi\left(\left\{\frac{x}{\varepsilon}\right\}\right)
\end{aligned}
$$

Using Proposition 3.2 we obtain

$$
\left\{\begin{array}{cl}
\mathcal{T}^{\varepsilon}\left(\partial_{\alpha} v_{\beta}^{\varepsilon}\right) \rightarrow v_{\beta}(x) \partial_{\alpha, y} w_{\beta}(y) & \text { strongly in } L^{2}\left(\Omega \times Y^{*}\right), \\
\mathcal{T}^{\varepsilon}\left(\partial_{\alpha \beta} v_{3}^{\varepsilon}\right) \rightarrow v_{3}(x) \partial_{\alpha \beta, y} w_{3}(y) & \text { strongly in } L^{2}\left(\Omega \times Y^{*}\right), \\
\mathcal{T}^{\varepsilon}\left(\partial_{\alpha} \psi^{\varepsilon}\right) \rightarrow \psi(x) \partial_{\alpha, y} \phi(y) & \text { strongly in } L^{2}\left(\Omega \times Y^{*}\right)
\end{array}\right.
$$

Let us set $\bar{v}_{i}(x, y)=v_{i}(x) w_{i}(y)$ and $\bar{\psi}(x, y)=\psi(x) \phi(y),(x, y) \in \Omega \times Y$. Then

$$
\left\{\begin{array}{rlrl}
\mathcal{T}^{\varepsilon}\left(\gamma_{\alpha \beta}\left(\boldsymbol{v}^{\varepsilon}\right)\right) & \rightarrow s_{\alpha \beta, y}(\overline{\boldsymbol{v}}) & & \text { strongly in } L^{2}\left(\Omega \times Y^{*}\right), \\
\mathcal{T}^{\varepsilon}\left(\rho_{\alpha \beta}\left(\boldsymbol{v}^{\varepsilon}\right)\right) & \rightarrow r_{\alpha \beta, y}(\overline{\boldsymbol{v}}) & & \text { strongly in } L^{2}\left(\Omega \times Y^{*}\right), \\
\mathcal{T}^{\varepsilon}\left(\nabla_{x} \psi^{\varepsilon}\right) & \rightarrow \nabla_{y} \bar{\psi} & \text { strongly in } L^{2}\left(\Omega \times Y^{*} ; \mathbb{R}^{2}\right)
\end{array}\right.
$$


With the same arguments as above we can pass to the limit in (8) to obtain

$$
\left\{\begin{array}{l}
\int_{\Omega \times Y^{*}}\left\{c_{M}^{\alpha \beta \sigma \tau}\left(\gamma_{\alpha \beta}(\boldsymbol{u})+s_{\alpha \beta, y}(\overline{\boldsymbol{u}})\right) s_{\sigma \tau, y}(\overline{\boldsymbol{v}})+\frac{t^{2}}{3} c_{F}^{\alpha \beta \sigma \tau}\left(\rho_{\alpha \beta}(\boldsymbol{u})+r_{\alpha \beta, y}(\overline{\boldsymbol{u}})\right) r_{\sigma \tau, y}(\overline{\boldsymbol{v}})\right\} \sqrt{a} \mathrm{~d} x \mathrm{~d} y \\
+\int_{\Omega \times Y^{*}} e^{\alpha \beta \sigma} s_{\alpha \beta, y}(\overline{\boldsymbol{v}})\left(\partial_{\sigma} \varphi+\partial_{\sigma, y} \bar{\varphi}\right) \sqrt{a} \mathrm{~d} x \mathrm{~d} y=0 \\
\int_{\Omega \times Y^{*}}\left\{-e^{\alpha \beta \sigma}\left(\gamma_{\alpha \beta}(\boldsymbol{u})+s_{\alpha \beta, y}(\overline{\boldsymbol{u}})\right) \partial_{\sigma, y} \bar{\psi}+d^{\alpha \beta}\left(\partial_{\alpha} \varphi+\partial_{\alpha, y} \bar{\varphi}\right) \partial_{\beta, y} \bar{\psi}\right\} \sqrt{a} \mathrm{~d} x \mathrm{~d} y=0
\end{array}\right.
$$

By density, (28) holds for all $(\overline{\boldsymbol{v}}, \bar{\psi}) \in \boldsymbol{L}^{2}\left(\Omega, \boldsymbol{V}_{\text {per }}\left(Y^{*}\right)\right) \times L^{2}\left(\Omega, H_{\text {per }}^{1}\left(Y^{*}\right)\right)$. Now, the variational problem $(20)$ is obtained by summating (26) and (28). We point out that (20) has a unique solution due to the symmetry and coercivity of the tensors $c_{M}, c_{F}, d, e$.

This completes the proof of Theorem 4.1.

We are now in position to write the local problems. First, in view of problem (28), we express the correctors $\overline{\boldsymbol{u}}$ and $\bar{\varphi}$ as a linear combination of basis functions $\left(\overline{\boldsymbol{g}}^{\lambda \mu}, \bar{\zeta}^{\lambda \mu}\right),\left(\overline{\boldsymbol{h}}^{\lambda \mu}, \bar{\theta}^{\lambda \mu}\right),\left(\overline{\boldsymbol{z}}^{\mu}, \bar{\eta}^{\mu}\right) \in \boldsymbol{L}^{2}\left(\Omega, \boldsymbol{V}_{\mathrm{per}}\left(Y^{*}\right)\right) \times$ $L^{2}\left(\Omega, H_{\mathrm{per}}^{1}\left(Y^{*}\right)\right)$ :

$$
\left\{\begin{array}{l}
\overline{\boldsymbol{u}}(x, y)=\gamma_{\lambda \mu}(\boldsymbol{u}(x)) \overline{\boldsymbol{g}}^{\lambda \mu}(x, y)+\rho_{\lambda \mu}(\boldsymbol{u}(x)) \overline{\boldsymbol{h}}^{\lambda \mu}(x, y)+\partial_{\mu} \varphi(x) \overline{\boldsymbol{z}}^{\mu}(x, y) \\
\bar{\varphi}(x, y)=\gamma_{\lambda \mu}(\boldsymbol{u}(x)) \bar{\zeta}^{\lambda \mu}(x, y)+\rho_{\lambda \mu}(\boldsymbol{u}(x)) \bar{\theta}^{\lambda \mu}(x, y)+\partial_{\mu} \varphi(x) \bar{\eta}^{\mu}(x, y)
\end{array}\right.
$$

From (28) and (29), the three pairs of local functions $\left(\overline{\boldsymbol{g}}^{\alpha \beta}, \bar{\zeta}^{\alpha \beta}\right),\left(\overline{\boldsymbol{h}}^{\alpha \beta}, \bar{\theta}^{\alpha \beta}\right)$ and $\left(\overline{\boldsymbol{z}}^{\sigma}, \bar{\eta}^{\sigma}\right)$ are given by the variational problems (30)-(32) below. Remark that the difference between problem (8) and problems (30)-(32) is that the operators $\gamma_{\alpha \beta}$ and $\rho_{\alpha \beta}$ in (8) have been replaced by $s_{\alpha \beta, y}$ and $r_{\alpha \beta, y}$ respectively:

$$
\begin{aligned}
& \begin{cases}\int_{Y^{*}}\left(c_{K, y}\left(\overline{\boldsymbol{g}}^{\alpha \beta}, \overline{\boldsymbol{v}}\right)+e_{y}\left(\overline{\boldsymbol{v}}, \bar{\zeta}^{\alpha \beta}\right)\right)=-\int_{Y^{*}} c_{M}^{\alpha \beta \sigma \tau} s_{\sigma \tau, y}(\overline{\boldsymbol{v}}) & \forall \overline{\boldsymbol{v}} \in \boldsymbol{V}_{\mathrm{per}}\left(Y^{*}\right), \\
\int_{Y^{*}}\left(-e_{y}\left(\overline{\boldsymbol{g}}^{\alpha \beta}, \bar{\psi}\right)+d_{y}\left(\bar{\zeta}^{\alpha \beta}, \bar{\psi}\right)\right)=\int_{Y^{*}} e^{\alpha \beta \sigma} \partial_{\sigma, y} \bar{\psi}, & \forall \bar{\psi} \in H_{\mathrm{per}}^{1}\left(Y^{*}\right),\end{cases} \\
& \begin{cases}\int_{Y^{*}}\left(c_{K, y}\left(\overline{\boldsymbol{h}}^{\alpha \beta}, \overline{\boldsymbol{v}}\right)+e_{y}\left(\overline{\boldsymbol{v}}, \bar{\theta}^{\alpha \beta}\right)\right)=-\frac{t^{2}}{3} \int_{Y^{*}} c_{F}^{\alpha \beta \sigma \tau} r_{\sigma \tau, y}(\overline{\boldsymbol{v}}), & \forall \overline{\boldsymbol{v}} \in \boldsymbol{V}_{\mathrm{per}}\left(Y^{*}\right), \\
\int_{Y^{*}}\left(-e_{y}\left(\overline{\boldsymbol{h}}^{\alpha \beta}, \bar{\psi}\right)+d_{y}\left(\bar{\theta}^{\alpha \beta}, \bar{\psi}\right)\right)=0 & \forall \bar{\psi} \in H_{\mathrm{per}}^{1}\left(Y^{*}\right),\end{cases} \\
& \begin{cases}\int_{Y^{*}}\left(c_{K, y}\left(\overline{\boldsymbol{z}}^{\sigma}, \overline{\boldsymbol{v}}\right)+e_{y}\left(\overline{\boldsymbol{v}}, \bar{\eta}^{\sigma}\right)\right)=-\int_{Y^{*}} e^{\alpha \beta \sigma} s_{\alpha \beta, y}(\overline{\boldsymbol{v}}) & \forall \overline{\boldsymbol{v}} \in \boldsymbol{V}_{\mathrm{per}}\left(Y^{*}\right), \\
\int_{Y^{*}}\left(-e_{y}\left(\overline{\boldsymbol{z}}^{\sigma}, \bar{\psi}\right)+d_{y}\left(\bar{\eta}^{\sigma}, \bar{\psi}\right)\right)=-\int_{Y^{*}} d^{\sigma \beta} \partial_{\beta, y} \bar{\psi} & \forall \bar{\psi} \in H_{\mathrm{per}}^{1}\left(Y^{*}\right),\end{cases}
\end{aligned}
$$

with the new notations

$$
\left\{\begin{aligned}
c_{K, y}(\boldsymbol{u}, \boldsymbol{v}) & =c_{M}^{\alpha \beta \sigma \tau} s_{\alpha \beta, y}(\boldsymbol{u}) s_{\sigma \tau, y}(\boldsymbol{v})+\frac{t^{2}}{3} c_{F}^{\alpha \beta \sigma \tau} r_{\alpha \beta, y}(\boldsymbol{u}) r_{\sigma \tau, y}(\boldsymbol{v}) \\
e_{y}(\boldsymbol{v}, \psi) & =e^{\alpha \beta \sigma} s_{\alpha \beta, y}(\boldsymbol{v}) \partial_{\sigma, y} \psi \\
d_{y}(\eta, \psi) & =d^{\alpha \beta} \partial_{\alpha, y} \eta \partial_{\beta, y} \psi
\end{aligned}\right.
$$

One can easily prove that problems (30)-(32) have a unique solution. We point out that the basis functions depend upon the macroscopic variable $x$ due to the definition of the operator $r_{\alpha \beta, y}$ and $c_{K, y}$.

Concerning the global homogenized problem we have the following result. 
Theorem 4.2. The limit elastic field $\boldsymbol{u} \in \boldsymbol{V}(\Omega)$ and the limit electric potential $\varphi \in H_{0}^{1}(\Omega)$ are the unique solution of the global homogenized variational problem

$$
\left\{\begin{array}{rlrl}
\int_{\Omega}\left(\bar{c}_{K}(\boldsymbol{u}, \boldsymbol{v})+\bar{e}_{K}(\boldsymbol{v}, \varphi)\right) \sqrt{a} \mathrm{~d} x & =\int_{\Omega} \boldsymbol{p} \cdot \boldsymbol{v} \sqrt{a} \mathrm{~d} x, & & \forall \boldsymbol{v} \in \boldsymbol{V}(\Omega), \\
\int_{\Omega}\left(-\bar{e}_{K}(\boldsymbol{u}, \psi)+\bar{d}_{K}(\varphi, \psi)\right) \sqrt{a} \mathrm{~d} x=0, & \forall \psi \in H_{0}^{1}(\Omega),
\end{array}\right.
$$

where $\bar{c}_{K}, \bar{e}_{K}, \bar{d}_{K}$ depend now on six new tensors given in (43) below.

Proof. By (29) we have

$$
\left\{\begin{aligned}
s_{\alpha \beta, y}(\overline{\boldsymbol{u}}) & =\gamma_{\lambda \mu}(\boldsymbol{u}) s_{\alpha \beta, y}\left(\overline{\boldsymbol{g}}^{\lambda \mu}\right)+\rho_{\lambda \mu}(\boldsymbol{u}) s_{\alpha \beta, y}\left(\overline{\boldsymbol{h}}^{\lambda \mu}\right)+\partial_{\mu} \varphi s_{\alpha \beta, y}\left(\overline{\boldsymbol{z}}^{\mu}\right) \\
r_{\alpha \beta, y}(\overline{\boldsymbol{u}}) & =\gamma_{\lambda \mu}(\boldsymbol{u}) r_{\alpha \beta, y}\left(\overline{\boldsymbol{g}}^{\lambda \mu}\right)+\rho_{\lambda \mu}(\boldsymbol{u}) r_{\alpha \beta, y}\left(\overline{\boldsymbol{h}}^{\lambda \mu}\right)+\partial_{\mu} \varphi r_{\alpha \beta, y}\left(\overline{\boldsymbol{z}}^{\mu}\right) \\
\partial_{\sigma, y} \bar{\varphi} & =\gamma_{\lambda \mu}(\boldsymbol{u}) \partial_{\sigma, y} \bar{\zeta}^{\lambda \mu}+\rho_{\lambda \mu}(\boldsymbol{u}) \partial_{\sigma, y} \bar{\theta}^{\lambda \mu}+\partial_{\mu} \varphi \partial_{\sigma, y} \bar{\eta}^{\mu}
\end{aligned}\right.
$$

Using the above relations, the first equation in (26) reads

$$
\begin{aligned}
& \int_{\Omega \times Y^{*}}\left\{\left[c_{M}^{\alpha \beta \sigma \tau}+c_{M}^{\sigma \tau \lambda \mu} s_{\lambda \mu, y}\left(\overline{\boldsymbol{g}}^{\alpha \beta}\right)+e^{\sigma \tau \lambda} \partial_{\lambda, y} \zeta^{\alpha \beta}\right] \gamma_{\alpha \beta}(\boldsymbol{u}) \gamma_{\sigma \tau}(\boldsymbol{v})+\frac{t^{2}}{3} c_{F}^{\sigma \tau \lambda \mu} r_{\lambda \mu, y}\left(\overline{\boldsymbol{g}}^{\alpha \beta}\right) \gamma_{\alpha \beta}(\boldsymbol{u}) \rho_{\sigma \tau}(\boldsymbol{v})\right. \\
& +\left[c_{M}^{\sigma \tau \lambda \mu} s_{\lambda \mu, y}\left(\overline{\boldsymbol{h}}^{\alpha \beta}\right)+e^{\alpha \beta \lambda} \partial_{\lambda, y} \bar{\theta}^{\alpha \beta}\right] \rho_{\alpha \beta}(\boldsymbol{u}) \gamma_{\lambda \mu}(\boldsymbol{v})+\frac{t^{2}}{3}\left[c_{F}^{\alpha \beta \sigma \tau}+c_{F}^{\sigma \tau \lambda \mu} r_{\lambda \mu, y}\left(\bar{\theta}^{\alpha \beta}\right)\right] \rho_{\alpha \beta}(\boldsymbol{u}) \rho_{\sigma \tau}(\boldsymbol{v}) \\
& \left.+\left[\left(e^{\alpha \beta \sigma}+e^{\alpha \beta \lambda} \partial_{\lambda, y} \bar{\eta}^{s}+c_{M}^{\alpha \beta \sigma \tau}\right) \gamma_{\alpha \beta}(\boldsymbol{u})+\frac{t^{2}}{3} c_{F}^{\alpha \beta \sigma \tau} r_{\lambda \mu, y}\left(\overline{\boldsymbol{z}}^{\sigma}\right) \rho_{\alpha \beta}(\boldsymbol{v})\right] \partial_{\sigma} \varphi\right\} \sqrt{a} \mathrm{~d} x \mathrm{~d} y=\left|Y^{*}\right| \int_{\Omega} \boldsymbol{p} \cdot \boldsymbol{v} \sqrt{a} \mathrm{~d} x
\end{aligned}
$$

Also, the second equation in (26) takes the form

$$
\begin{aligned}
& \int_{\Omega \times Y^{*}}\left\{\left[-e^{\alpha \beta \sigma}-e^{\lambda \mu \sigma} s_{\lambda \mu, y}\left(\overline{\boldsymbol{g}}^{\alpha \beta}\right)+d^{\lambda \sigma} \partial_{\lambda, y} \bar{\zeta}^{\alpha \beta}\right] \gamma_{\alpha \beta}(\boldsymbol{v}) \partial_{\sigma} \psi+\left[-e^{\lambda \mu \sigma} s_{\lambda \mu, y}\left(\overline{\boldsymbol{h}}^{\alpha \beta}\right)+d^{\lambda \sigma} \partial_{\lambda, y} \bar{\theta}^{\alpha \beta}\right] \rho_{\alpha \beta}(\boldsymbol{v}) \partial_{\sigma} \psi\right. \\
& \left.+\left[d^{\alpha \beta}+d^{\alpha \lambda} \partial_{\lambda, y} \bar{\eta}^{\beta}-e^{\lambda \mu \alpha} s_{\lambda \mu, y}\left(\overline{\boldsymbol{z}}^{\beta}\right)\right] \partial_{\alpha} \varphi \partial_{\beta} \psi\right\} \sqrt{a} \mathrm{~d} x \mathrm{~d} y=0
\end{aligned}
$$

Let us introduce the notations

$$
\left\{\begin{array}{l}
\bar{c}_{M}^{\alpha \beta \sigma \tau}(x)=\frac{1}{\left|Y^{*}\right|} \int_{Y^{*}} c_{M}^{\sigma \tau \lambda \mu}\left(\delta_{\lambda}^{\alpha} \delta_{\mu}^{\beta}+s_{\lambda \mu, y}\left(\overline{\boldsymbol{g}}^{\alpha \beta}\right)\right)+e^{\sigma \tau \lambda} \partial_{\lambda, y} \bar{\zeta}^{\alpha \beta} \\
\bar{c}_{F}^{\alpha \beta \sigma \tau}(x)=\frac{1}{\left|Y^{*}\right|} \int_{Y^{*}} c_{F}^{\sigma \tau \lambda \mu}\left(\delta_{\lambda}^{\alpha} \delta_{\mu}^{\beta}+r_{\lambda \mu, y}\left(\overline{\boldsymbol{h}}^{\alpha \beta}\right)\right) \\
\bar{c}_{1}^{\alpha \beta \sigma \tau}(x)=\frac{1}{\left|Y^{*}\right|} \int_{Y^{*}} c_{F}^{\sigma \tau \lambda \mu} r_{\lambda \mu, y}\left(\overline{\boldsymbol{g}}^{\alpha \beta}\right), \\
\bar{c}_{2}^{\alpha \beta \sigma \tau}(x)=\frac{1}{\left|Y^{*}\right|} \int_{Y^{*}} c_{M}^{\sigma \tau \lambda \mu} s_{\lambda \mu, y}\left(\overline{\boldsymbol{h}}^{\alpha \beta}\right)+e^{\sigma \tau \lambda} \partial_{\lambda, y} \bar{\theta}^{\alpha \beta} \\
\bar{e}^{\alpha \beta \sigma}(x)=\frac{1}{\left|Y^{*}\right|} \int_{Y^{*}} e^{\alpha \beta \lambda}\left(\delta_{\lambda}^{\sigma}+\partial_{\lambda, y} \bar{\eta}^{\sigma}\right)+c_{M}^{\alpha \beta \lambda \mu} s_{\lambda \mu, y}\left(\overline{\boldsymbol{z}}^{\sigma}\right), \\
\bar{l}^{\alpha \beta \sigma}(x)=\frac{1}{\left|Y^{*}\right|} \int_{Y^{*}} c_{F}^{\alpha \beta \lambda \mu} r_{\lambda \mu, y}\left(\overline{\boldsymbol{z}}^{\sigma}\right), \\
\bar{f}^{\alpha \beta \sigma}(x)=\frac{1}{\left|Y^{*}\right|} \int_{Y^{*}} e^{\lambda \mu \sigma}\left(\delta_{\lambda}^{\alpha} \delta_{\mu}^{\beta}+s_{\lambda \mu, y}\left(\overline{\boldsymbol{g}}^{\alpha \beta}\right)\right)-d^{\lambda \sigma} \partial_{\lambda, y} \bar{\zeta}^{\alpha \beta} \\
\bar{q}^{\alpha \beta \sigma}(x)=\frac{1}{\left|Y^{*}\right|} \int_{Y^{*}} e^{\lambda \mu \sigma} s_{\lambda \mu, y}\left(\overline{\boldsymbol{h}}^{\alpha \beta}\right)-d^{\lambda \sigma} \partial_{\lambda, y} \bar{\theta}^{\alpha \beta} \\
\bar{d}^{\alpha \beta}(x)=\frac{1}{\left|Y^{*}\right|} \int_{Y^{*}}\left(-e^{\lambda \mu \alpha} s_{\lambda \mu, y}\left(\overline{\boldsymbol{z}}^{\beta}\right)+d^{\alpha \lambda}\left(\delta_{\lambda}^{\beta}+\partial_{\lambda, y} \bar{\eta}^{\beta}\right)\right) .
\end{array}\right.
$$


From (35)-(37) we get the following global variational problem

$$
\left\{\begin{aligned}
\int_{\Omega}\left(\bar{c}_{K}(\boldsymbol{u}, \boldsymbol{v})+\bar{e}_{K}(\boldsymbol{v}, \varphi)\right) \sqrt{a} \mathrm{~d} x & =\int_{\Omega} \boldsymbol{p} \cdot \boldsymbol{v} \sqrt{a} \mathrm{~d} x, & & \forall \boldsymbol{v} \in \boldsymbol{V}(\Omega), \\
\int_{\Omega}\left(-\bar{f}_{K}(\boldsymbol{u}, \psi)+\bar{d}_{K}(\varphi, \psi)\right) \sqrt{a} \mathrm{~d} x & =0, & & \forall \psi \in H_{0}^{1}(\Omega),
\end{aligned}\right.
$$

where

$$
\left\{\begin{array}{l}
\bar{c}_{K}(\boldsymbol{u}, \boldsymbol{v})=\bar{c}_{M}^{\alpha \beta \sigma \tau} \gamma_{\alpha \beta}(\boldsymbol{u}) \gamma_{\sigma \tau}(\boldsymbol{v})+\frac{t^{2}}{3} \bar{c}_{F}^{\alpha \beta \sigma \tau} \rho_{\alpha \beta}(\boldsymbol{u}) \rho_{\sigma \tau}(\boldsymbol{v})+\frac{t^{2}}{3} \bar{c}_{1}^{\alpha \beta \sigma \tau} \gamma_{\alpha \beta}(\boldsymbol{u}) \rho_{\sigma \tau}(\boldsymbol{v})+\bar{c}_{2}^{\alpha \beta \sigma \tau} \rho_{\alpha \beta}(\boldsymbol{u}) \gamma_{\sigma \tau}(\boldsymbol{v}), \\
\bar{e}_{K}(\boldsymbol{v}, \psi)=\bar{e}^{\alpha \beta \sigma} \gamma_{\alpha \beta}(\boldsymbol{v}) \partial_{\sigma} \psi+\frac{t^{2}}{3} \bar{l}^{\alpha \beta \sigma} \rho_{\alpha \beta}(\boldsymbol{v}) \partial_{\sigma} \psi \\
\bar{f}_{K}(\boldsymbol{v}, \psi)=\bar{f}^{\alpha \beta \sigma} \gamma_{\alpha \beta}(\boldsymbol{v}) \partial_{\sigma} \psi+\bar{q}^{\alpha \beta \sigma} \rho_{\alpha \beta}(\boldsymbol{v}) \partial_{\sigma} \psi \\
\bar{d}_{K}(\varphi, \psi)=\bar{d}^{\alpha \beta} \partial_{\alpha} \varphi \partial_{\beta} \psi
\end{array}\right.
$$

The following result asserts the symmetry of the tensors $\bar{c}_{M}, \bar{c}_{F}, \bar{d}$.

Lemma 4.3. We have
(i) $\frac{t^{2}}{3} \bar{c}_{1}^{\alpha \beta \sigma \tau}=\bar{c}_{2}^{\sigma \tau \alpha \beta}$.
(ii) $\bar{f}^{\alpha \beta \sigma}=\bar{f}^{\beta \alpha \sigma}=\bar{e}^{\alpha \beta \sigma}$ and $\bar{q}^{\alpha \beta \sigma}=\bar{q}^{\beta \alpha \sigma}=\frac{t^{2}}{3} \bar{l}^{\alpha \beta \sigma}$.
(iii) $\bar{c}_{M}^{\alpha \beta \sigma \tau}=\bar{c}_{M}^{\sigma \tau \alpha \beta}$ and $\bar{c}_{F}^{\alpha \beta \sigma \tau}=\bar{c}_{F}^{\sigma \tau \alpha \beta}$.
(iv) $\bar{d}^{\alpha \beta}=\bar{d}^{\beta \alpha}$.

Proof. The main idea is to choose particular test functions $(\overline{\boldsymbol{v}}, \bar{\psi})$ in the variational problems (30)-(32).

(i) Letting $(\overline{\boldsymbol{v}}, \bar{\psi})=\left(\overline{\boldsymbol{g}}^{\alpha \beta}, \bar{\zeta}^{\alpha \beta}\right)$ in (31) we obtain

$$
\left\{\begin{array}{l}
\int_{Y^{*}}\left(c_{K, y}\left(\overline{\boldsymbol{h}}^{\sigma \tau}, \overline{\boldsymbol{g}}^{\alpha \beta}\right)+e_{y}\left(\overline{\boldsymbol{g}}^{\alpha \beta}, \bar{\theta}^{\sigma \tau}\right)\right)=-\frac{t^{2}}{3} \int_{Y^{*}} c_{F}^{\sigma \tau \lambda \mu} r_{\lambda \mu, y}\left(\overline{\boldsymbol{g}}^{\alpha \beta}\right), \\
\int_{Y^{*}}\left(-e_{y}\left(\overline{\boldsymbol{h}}^{\sigma \tau}, \bar{\zeta}^{\alpha \beta}\right)+d_{y}\left(\bar{\theta}^{\sigma \tau}, \bar{\zeta}^{\alpha \beta}\right)\right)=0 .
\end{array}\right.
$$

By subtraction, the above relations yield

$$
\frac{t^{2}}{3} \int_{Y^{*}} c_{F}^{\sigma \tau \lambda \mu} r_{\lambda \mu, y}\left(\overline{\boldsymbol{g}}^{\alpha \beta}\right)=-\int_{Y^{*}} c_{K, y}\left(\overline{\boldsymbol{h}}^{\sigma \tau}, \overline{\boldsymbol{g}}^{\alpha \beta}\right)+e_{y}\left(\overline{\boldsymbol{g}}^{\alpha \beta}, \bar{\theta}^{\sigma \tau}\right)+e_{y}\left(\overline{\boldsymbol{h}}^{\sigma \tau}, \bar{\zeta}^{\alpha \beta}\right)-d_{y}\left(\bar{\theta}^{\sigma \tau}, \bar{\zeta}^{\alpha \beta}\right) .
$$

Hence

$$
\begin{aligned}
\frac{t^{2}}{3} \bar{c}_{1}^{\alpha \beta \sigma \tau} & =\frac{t^{2}}{3\left|Y^{*}\right|} \int_{Y^{*}} c_{F}^{\sigma \tau \lambda \mu} r_{\lambda \mu, y}\left(\overline{\boldsymbol{g}}^{\alpha \beta}\right) \\
& =-\frac{1}{\left|Y^{*}\right|} \int_{Y^{*}} c_{K, y}\left(\overline{\boldsymbol{h}}^{\sigma \tau}, \overline{\boldsymbol{g}}^{\alpha \beta}\right)+e_{y}\left(\overline{\boldsymbol{g}}^{\alpha \beta}, \bar{\theta}^{\sigma \tau}\right)+e_{y}\left(\overline{\boldsymbol{h}}^{\sigma \tau}, \bar{\zeta}^{\alpha \beta}\right)-d_{y}\left(\bar{\theta}^{\sigma \tau}, \bar{\zeta}^{\alpha \beta}\right) .
\end{aligned}
$$

Taking $(\overline{\boldsymbol{v}}, \bar{\psi})=\left(\overline{\boldsymbol{h}}^{\sigma \tau}, \bar{\theta}^{\sigma \tau}\right)$ in (30) and then subtracting, we find

$$
\begin{aligned}
\bar{c}_{2}^{\alpha \beta \sigma \tau} & =\frac{1}{\left|Y^{*}\right|} \int_{Y^{*}} c_{M}^{\sigma \tau \lambda \mu} s_{\lambda \mu, y}\left(\overline{\boldsymbol{h}}^{\alpha \beta}\right)+e^{\sigma \tau \lambda} \partial_{\lambda, y} \bar{\theta}^{\alpha \beta} \\
& =-\frac{1}{\left|Y^{*}\right|} \int_{Y^{*}} c_{K, y}\left(\overline{\boldsymbol{g}}^{\alpha \beta}, \overline{\boldsymbol{h}}^{\sigma \tau}\right)+e_{y}\left(\overline{\boldsymbol{h}}^{\sigma \tau}, \bar{\zeta}^{\alpha \beta}\right)+e_{y}\left(\overline{\boldsymbol{g}}^{\alpha \beta}, \bar{\theta}^{\sigma \tau}\right)-d_{y}\left(\bar{\zeta}^{\alpha \beta}, \bar{\theta}^{\sigma \tau}\right) .
\end{aligned}
$$


From (39) and (40) we get $\frac{t^{2}}{3} \bar{c}_{1}^{\alpha \beta \sigma \tau}=\bar{c}_{2}^{\alpha \beta \sigma \tau}$.

(ii) Let us take $(\overline{\boldsymbol{v}}, \bar{\psi})=\left(\overline{\boldsymbol{g}}^{\alpha \beta}, \bar{\zeta}^{\alpha \beta}\right)$ as test functions in (32). We get

$$
\left\{\begin{array}{l}
\int_{Y^{*}}\left(c_{K, y}\left(\overline{\boldsymbol{z}}^{\sigma}, \overline{\boldsymbol{g}}^{\alpha \beta}\right)+e_{y}\left(\overline{\boldsymbol{g}}^{\alpha \beta}, \bar{\eta}^{\sigma}\right)=-\int_{Y^{*}} e^{\lambda \mu \sigma} s_{\lambda \mu, y}\left(\overline{\boldsymbol{g}}^{\alpha \beta}\right),\right. \\
\int_{Y^{*}}\left(-e_{y}\left(\overline{\boldsymbol{z}}^{\sigma}, \bar{\zeta}^{\alpha \beta}\right)+d_{y}\left(\bar{\eta}^{\sigma}, \bar{\zeta}^{\alpha \beta}\right)\right)=-\int_{Y^{*}} d^{\lambda \sigma} \partial_{\lambda, y} \bar{\zeta}^{\alpha \beta} .
\end{array}\right.
$$

Subtracting the above equalities we obtain

$$
-\int_{Y^{*}} e^{\lambda \mu \sigma}\left(s_{\lambda \mu, y}\left(\overline{\boldsymbol{g}}^{\alpha \beta}\right)-\partial_{\lambda, y} \bar{\zeta}^{\alpha \beta}\right)=\int_{Y^{*}} c_{K, y}\left(\overline{\boldsymbol{g}}^{\alpha \beta}, \overline{\boldsymbol{z}}^{\sigma}\right)+e_{y}\left(\overline{\boldsymbol{g}}^{\alpha \beta}, \bar{\eta}^{\sigma}\right)+e_{y}\left(\overline{\boldsymbol{z}}^{\sigma}, \bar{\zeta}^{\alpha \beta}\right)-d_{y}\left(\bar{\eta}^{\sigma}, \bar{\zeta}^{\alpha \beta}\right) .
$$

Furthermore, by (37) we deduce

$$
\begin{aligned}
\bar{f}^{\alpha \beta \sigma} & =\bar{e}^{\alpha \beta \sigma}+\int_{Y^{*}} e^{\lambda \mu \sigma}\left(s_{\lambda \mu, y}\left(\overline{\boldsymbol{g}}^{\alpha \beta}\right)-\partial_{\lambda, y} \bar{\zeta}^{\alpha \beta}\right) \\
& =\bar{e}^{\alpha \beta \sigma}-\frac{1}{\left|Y^{*}\right|} \int_{Y^{*}} c_{K, y}\left(\overline{\boldsymbol{g}}^{\alpha \beta}, \overline{\boldsymbol{z}}^{\sigma}\right)+e_{y}\left(\overline{\boldsymbol{g}}^{\alpha \beta}, \bar{\eta}^{\sigma}\right)+e_{y}\left(\overline{\boldsymbol{z}}^{\sigma}, \bar{\zeta}^{\alpha \beta}\right)-d_{y}\left(\bar{\eta}^{\sigma}, \bar{\zeta}^{\alpha \beta}\right)
\end{aligned}
$$

Hence $\bar{f}^{\alpha \beta \sigma}=\bar{f}^{\beta \alpha \sigma}$. Taking $(\overline{\boldsymbol{v}}, \bar{\psi})=\left(\overline{\boldsymbol{z}}^{\sigma}, \bar{\eta}^{\sigma}\right)$ in (30), by combination we have

$$
-\int_{Y^{*}} c_{M}^{\alpha \beta \sigma \tau}\left(s_{\sigma \tau, y}\left(\overline{\boldsymbol{z}}^{\sigma}\right)+\partial_{\lambda, y} \bar{\eta}^{\sigma}\right)=\int_{Y^{*}} c_{K, y}\left(\overline{\boldsymbol{g}}^{\alpha \beta}, \overline{\boldsymbol{z}}^{\sigma}\right)+e_{y}\left(\overline{\boldsymbol{g}}^{\alpha \beta}, \bar{\eta}^{\sigma}\right)+e_{y}\left(\overline{\boldsymbol{z}}^{\sigma}, \bar{\zeta}^{\alpha \beta}\right)-d_{y}\left(\bar{\eta}^{\sigma}, \bar{\zeta}^{\alpha \beta}\right) .
$$

By (37), (41) and the above equality we obtain

$$
\begin{aligned}
\bar{e}^{\alpha \beta \sigma} & =\bar{e}^{\alpha \beta \sigma}+\int_{Y^{*}} \int_{Y^{*}} c_{M}^{\alpha \beta \sigma \tau}\left(s_{\sigma \tau, y}\left(\overline{\boldsymbol{z}}^{\sigma}\right)+\partial_{\lambda, y} \bar{\eta}^{\sigma}\right) \\
& =\bar{e}^{\alpha \beta \sigma}-\frac{1}{\left|Y^{*}\right|} \int_{Y^{*}} c_{K, y}\left(\overline{\boldsymbol{g}}^{\alpha \beta}, \overline{\boldsymbol{z}}^{\sigma}\right)+e_{y}\left(\overline{\boldsymbol{g}}^{\alpha \beta}, \bar{\eta}^{\sigma}\right)+e_{y}\left(\overline{\boldsymbol{z}}^{\sigma}, \bar{\zeta}^{\alpha \beta}\right)-d_{y}\left(\bar{\eta}^{\sigma}, \bar{\zeta}^{\alpha \beta}\right) . \\
& =\bar{f}^{\alpha \beta \sigma}
\end{aligned}
$$

We have proved that $\bar{f}^{\alpha \beta \sigma}=\bar{f}^{\beta \alpha \sigma}=\bar{e}^{\alpha \beta \sigma}$. In the same manner we obtain $\bar{q}^{\alpha \beta \sigma}=\bar{q}^{\beta \alpha \sigma}=\frac{t^{2}}{3} \bar{l}^{\alpha \beta \sigma}$.

(iii) First, from (37) we have

$$
\bar{c}_{M}^{\alpha \beta \sigma \tau}(x)=c_{M}^{\alpha \beta \sigma \tau}+\frac{1}{\left|Y^{*}\right|} \int_{Y^{*}} c_{M}^{\alpha \beta \lambda \mu} s_{\lambda \mu, y}\left(\overline{\boldsymbol{g}}^{\sigma \tau}\right)+e^{\alpha \beta \lambda} \partial_{\lambda, y} \bar{\zeta}^{\sigma \tau} .
$$

We choose $(\overline{\boldsymbol{v}}, \bar{\psi})=\left(\overline{\boldsymbol{g}}^{\sigma \tau}, \bar{\zeta}^{\sigma \tau}\right)$ as test functions in (30). We get

$$
\left\{\begin{array}{l}
\int_{Y^{*}}\left(c_{K, y}\left(\overline{\boldsymbol{g}}^{\alpha \beta}, \overline{\boldsymbol{g}}^{\sigma \tau}\right)+e_{y}\left(\overline{\boldsymbol{g}}^{\sigma \tau}, \bar{\zeta}^{\alpha \beta}\right)\right)=-\int_{Y^{*}} c_{M}^{\alpha \beta \lambda \mu} s_{\lambda \mu, y}\left(\overline{\boldsymbol{g}}^{\sigma \tau}\right), \\
\int_{Y^{*}}\left(-e_{y}\left(\overline{\boldsymbol{g}}^{\alpha \beta}, \bar{\zeta}^{\sigma \tau}\right)+d_{y}\left(\bar{\zeta}^{\alpha \beta}, \bar{\zeta}^{\sigma \tau}\right)\right)=0 .
\end{array}\right.
$$

Hence

$$
\bar{c}_{M}^{\alpha \beta \sigma \tau}=c_{M}^{\alpha \beta \sigma \tau}-\frac{1}{\left|Y^{*}\right|} \int_{Y^{*}} c_{K, y}\left(\overline{\boldsymbol{g}}^{\alpha \beta}, \overline{\boldsymbol{g}}^{\sigma \tau}\right)+e_{y}\left(\overline{\boldsymbol{g}}^{\sigma \tau}, \bar{\zeta}^{\alpha \beta}\right)+e_{y}\left(\overline{\boldsymbol{g}}^{\alpha \beta}, \bar{\zeta}^{\sigma \tau}\right)-d_{y}\left(\bar{\zeta}^{\alpha \beta}, \bar{\zeta}^{\sigma \tau}\right)
$$


It follows that $\bar{c}_{M}^{\alpha \beta \sigma \tau}=\bar{c}_{M}^{\sigma \tau \alpha \beta}$. In the same manner, by taking $(\overline{\boldsymbol{v}}, \bar{\psi})=\left(\overline{\boldsymbol{h}}^{\sigma \tau}, \bar{\theta}^{\sigma \tau}\right)$ in (31), we deduce $\bar{c}_{F}^{\alpha \beta \sigma \tau}=$ $\bar{c}_{F}^{\sigma \tau \alpha \beta}$.

(iv) With the same idea as above, we consider $(\overline{\boldsymbol{v}}, \bar{\psi})=\left(\overline{\boldsymbol{z}}^{\sigma}, \bar{\eta}^{\sigma}\right)$ in $(32)$ in order to get

$$
\bar{d}^{\alpha \beta}=d^{\alpha \beta}-\frac{1}{\left|Y^{*}\right|} \int_{Y^{*}} c_{K, y}\left(\overline{\boldsymbol{z}}^{\alpha}, \overline{\boldsymbol{z}}^{\beta}\right)+e_{y}\left(\overline{\boldsymbol{z}}^{\alpha}, \bar{\eta}^{\beta}\right)+e_{y}\left(\overline{\boldsymbol{z}}^{\beta}, \bar{\eta}^{\alpha}\right)-d_{y}\left(\bar{\eta}^{\alpha}, \bar{\eta}^{\beta}\right)=\bar{d}^{\beta \alpha}
$$

The proof of Lemma 4.3 is now complete.

From (37) we obtain the following expressions of homogenized tensors $\bar{c}_{M}, \bar{c}_{F}, \bar{e}, \bar{d}$ and of the new flexural coupling tensors $\bar{c}, \bar{l}$ :

$$
\left\{\begin{array}{l}
\bar{c}_{M}^{\alpha \beta \sigma \tau}(x)=\frac{1}{\left|Y^{*}\right|} \int_{Y^{*}} c_{M}^{\alpha \beta \lambda \mu}\left(\delta_{\lambda}^{\sigma} \delta_{\mu}^{\tau}+s_{\lambda \mu, y}\left(\overline{\boldsymbol{g}}^{\sigma \tau}\right)\right)+e^{\alpha \beta \lambda} \partial_{\lambda, y} \bar{\zeta}^{\sigma \tau} \\
\bar{c}_{F}^{\alpha \beta \sigma \tau}(x)=\frac{1}{\left|Y^{*}\right|} \int_{Y^{*}} c_{F}^{\alpha \beta \lambda \mu}\left(\delta_{\lambda}^{\sigma} \delta_{\mu}^{\tau}+r_{\lambda \mu, y}\left(\overline{\boldsymbol{h}}^{\sigma \tau}\right)\right) \\
\bar{c}^{\alpha \beta \sigma \tau}(x)=\frac{1}{\left|Y^{*}\right|} \int_{Y^{*}} c_{F}^{\alpha \beta \lambda \mu} r_{\lambda \mu, y}\left(\overline{\boldsymbol{g}}^{\sigma \tau}\right) \\
\bar{e}^{\alpha \beta \sigma}(x)=\frac{1}{\left|Y^{*}\right|} \int_{Y^{*}} e^{\alpha \beta \lambda}\left(\delta_{\lambda}^{\sigma}+\partial_{\lambda, y} \bar{\eta}^{\sigma}\right)+c_{M}^{\alpha \beta \lambda \mu} s_{\lambda \mu, y}\left(\overline{\boldsymbol{z}}^{\sigma}\right), \\
\bar{l}^{\alpha \beta \sigma}(x)=\frac{1}{\left|Y^{*}\right|} \int_{Y^{*}} c_{F}^{\alpha \beta \lambda \mu} r_{\lambda \mu, y}\left(\overline{\boldsymbol{z}}^{\sigma}\right), \\
\bar{d}^{\alpha \beta}(x)=\frac{1}{\left|Y^{*}\right|} \int_{Y^{*}}\left(-e^{\lambda \mu \alpha} s_{\lambda \mu, y}\left(\overline{\boldsymbol{z}}^{\beta}\right)+d^{\alpha \lambda}\left(\delta_{\lambda}^{\beta}+\partial_{\lambda, y} \bar{\eta}^{\beta}\right)\right) .
\end{array}\right.
$$

Remark also that, by virtue of Lemma 4.3, problem (38) rewrites

$$
\left\{\begin{array}{rlrl}
\int_{\Omega}\left(\bar{c}_{K}(\boldsymbol{u}, \boldsymbol{v})+\bar{e}_{K}(\boldsymbol{v}, \varphi)\right) \sqrt{a} \mathrm{~d} x=\int_{\Omega} \boldsymbol{p} \cdot \boldsymbol{v} \sqrt{a} \mathrm{~d} x & & \forall \boldsymbol{v} \in \boldsymbol{V}(\Omega), \\
\int_{\Omega}\left(-\bar{e}_{K}(\boldsymbol{u}, \psi)+\bar{d}_{K}(\varphi, \psi)\right) \sqrt{a} \mathrm{~d} x=0 & \forall \psi \in H_{0}^{1}(\Omega),
\end{array}\right.
$$

where

$$
\left\{\begin{array}{l}
\bar{c}_{K}(\boldsymbol{u}, \boldsymbol{v})=\bar{c}_{M}^{\alpha \beta \sigma \tau} \gamma_{\alpha \beta}(\boldsymbol{u}) \gamma_{\sigma \tau}(\boldsymbol{v})+\frac{t^{2}}{3} \bar{c}_{F}^{\alpha \beta \sigma \tau} \rho_{\alpha \beta}(\boldsymbol{u}) \rho_{\sigma \tau}(\boldsymbol{v})+\frac{t^{2}}{3} \bar{c}^{\alpha \beta \sigma \tau} \gamma_{\alpha \beta}(\boldsymbol{u}) \rho_{\sigma \tau}(\boldsymbol{v})+\frac{t^{2}}{3} \bar{c}^{\sigma \tau \alpha \beta} \rho_{\alpha \beta}(\boldsymbol{u}) \gamma_{\sigma \tau}(\boldsymbol{v}) \\
\bar{e}_{K}(\boldsymbol{v}, \psi)=\bar{e}^{\alpha \beta \sigma} \gamma_{\alpha \beta}(\boldsymbol{v}) \partial_{\sigma} \psi+\frac{t^{2}}{3} \bar{l}^{\alpha \beta \sigma} \rho_{\alpha \beta}(\boldsymbol{v}) \partial_{\sigma} \psi \\
\bar{d}_{K}(\varphi, \psi)=\bar{d}^{\alpha \beta} \partial_{\alpha} \varphi \partial_{\beta} \psi
\end{array}\right.
$$

It remains only to prove the existence and the uniqueness of the solution corresponding to the limit problem (34). This follows via Lax-Milgram theorem by showing the coercivity of the tensors $\left(\bar{c}_{M}^{\alpha \beta \sigma \tau}\right),\left(\bar{c}_{F}^{\alpha \beta \sigma \tau}\right)$ and $\left(\bar{d}^{\alpha \beta}\right)$. Note that by virtue of Lemma 4.3 all these tensors are symmetric. Let us first prove the coercivity of $\left(\bar{c}_{M}^{\alpha \beta \sigma \tau}\right)$. To this aim, consider a symmetric tensor $X=\left(X_{\alpha \beta}\right)$. Then, from (42) we have

$$
\bar{c}_{M}^{\alpha \beta \sigma \tau} X_{\alpha \beta} X_{\sigma \tau}=c_{M}^{\alpha \beta \sigma \tau} X_{\alpha \beta} X_{\sigma \tau}+\frac{1}{\left|Y^{*}\right|} \int_{Y^{*}} c_{M}^{\alpha \beta \lambda \mu} s_{\lambda \mu, y}\left(\overline{\boldsymbol{g}}^{\sigma \tau} X_{\alpha \beta}\right) X_{\sigma \tau}+e^{\alpha \beta \lambda} \partial_{\lambda, y}\left(\bar{\zeta}^{\sigma \tau} X_{\sigma \tau}\right) X_{\alpha \beta} .
$$

Moreover, by $(30)$ we deduce that $(\overline{\boldsymbol{w}}, \bar{\xi})=\left(\overline{\boldsymbol{g}}^{\sigma \tau} X_{\sigma \tau}, \bar{\zeta}^{\sigma \tau} X_{\sigma \tau}\right)$ is solution of the variational problem

$$
\begin{cases}\int_{Y^{*}}\left(c_{K, y}(\overline{\boldsymbol{w}}, \overline{\boldsymbol{v}})+e_{y}(\overline{\boldsymbol{v}}, \bar{\xi})\right)=-\int_{Y^{*}} c_{M}^{\alpha \beta \lambda \mu} s_{\lambda \mu, y}(\overline{\boldsymbol{v}}) X_{\alpha \beta}, & \forall \overline{\boldsymbol{v}} \in \boldsymbol{V}_{\mathrm{per}}\left(Y^{*}\right), \\ \int_{Y^{*}}\left(-e_{y}(\overline{\boldsymbol{w}}, \bar{\psi})+d_{y}(\bar{\xi}, \bar{\psi})=\int_{Y^{*}} e^{\alpha \beta \sigma} \partial_{\sigma, y} \bar{\psi} X_{\alpha \beta},\right. & \forall \bar{\psi} \in H_{\mathrm{per}}^{1}\left(Y^{*}\right) .\end{cases}
$$


Therefore $(\overline{\boldsymbol{w}}, \bar{\xi})$ is a saddle point associated to the functional

$$
I: \boldsymbol{V}_{\mathrm{per}}\left(Y^{*}\right) \times H_{\mathrm{per}}^{1}\left(Y^{*}\right) \rightarrow \mathbb{R}
$$

defined by

$$
\begin{aligned}
I(\overline{\boldsymbol{v}}, \bar{\psi})= & \frac{1}{2} \int_{Y^{*}} c_{M}^{\alpha \beta \sigma \tau}\left(s_{\alpha \beta, y}(\overline{\boldsymbol{v}})+X_{\alpha \beta}\right)\left(s_{\sigma \tau, y}(\overline{\boldsymbol{v}})+X_{\sigma \tau}\right) \sqrt{a} \mathrm{~d} y+\frac{t^{2}}{6} \int_{Y^{*}} c_{F}^{\alpha \beta \sigma \tau} r_{\alpha \beta, y}(\overline{\boldsymbol{v}}) r_{\sigma \tau, y}(\overline{\boldsymbol{v}}) \sqrt{a} \mathrm{~d} y \\
& +\int_{Y^{*}} e^{\alpha \beta \sigma}\left(s_{\alpha \beta, y}(\overline{\boldsymbol{v}})+X_{\alpha \beta}\right) \partial_{\lambda, y} \bar{\psi} \sqrt{a} \mathrm{~d} y-\frac{1}{2} \int_{Y^{*}} d^{\alpha \beta} \partial_{\alpha, y} \bar{\psi} \partial_{\beta, y} \bar{\psi} \sqrt{a} \mathrm{~d} y .
\end{aligned}
$$

This yields

$$
I(\overline{\boldsymbol{w}}, \bar{\psi}) \leq I(\overline{\boldsymbol{w}}, \bar{\xi}) \leq I(\overline{\boldsymbol{v}}, \bar{\xi}), \quad \text { for all }(\overline{\boldsymbol{v}}, \bar{\psi}) \in \boldsymbol{V}_{\mathrm{per}}\left(Y^{*}\right) \times H_{\mathrm{per}}^{1}\left(Y^{*}\right) .
$$

Consequently, for $\bar{\psi}=0$ we get

$$
\begin{aligned}
I(\overline{\boldsymbol{w}}, \bar{\xi}) & \geq I(\overline{\boldsymbol{w}}, 0) \\
& =\frac{1}{2} \int_{Y^{*}} c_{M}^{\alpha \beta \lambda \mu}\left(s_{\alpha \beta, y}(\overline{\boldsymbol{w}})+X_{\alpha \beta}\right)\left(s_{\lambda \mu, y}(\overline{\boldsymbol{w}})+X_{\lambda \mu}\right) \sqrt{a} \mathrm{~d} y+\frac{t^{2}}{6} \int_{Y^{*}} c_{F}^{\alpha \beta \sigma \tau} r_{\alpha \beta, y}(\overline{\boldsymbol{w}}) r_{\sigma \tau, y}(\overline{\boldsymbol{w}}) \sqrt{a} \mathrm{~d} y \\
& >0
\end{aligned}
$$

Moreover, taking $(\overline{\boldsymbol{v}}, \bar{\psi})=(\overline{\boldsymbol{w}}, \bar{\xi})$ in $(44)$ we obtain

$$
\bar{c}_{M}^{\alpha \beta \tau \sigma} X_{\alpha \beta} X_{\sigma \tau}=2 I(\overline{\boldsymbol{w}}, \bar{\xi})>0 .
$$

Set $\mathcal{B}=\left\{X=\left(X_{\alpha \beta}\right) ; X\right.$ is symmetric and $\left.X_{\alpha \beta} X_{\alpha \beta}=1\right\}$ and consider $\Psi: \mathcal{B} \rightarrow \mathbb{R}$ defined by

$$
\Psi\left(X_{\alpha \beta}\right)=\bar{c}_{M}^{\alpha \beta \rho \sigma} X_{\alpha \beta} X_{\rho \sigma} .
$$

It is easy to see that $\Psi$ is continuous on $\mathcal{B}$ endowed with the standard topology defined by the norm $\|X\|=$ $\left(X_{\alpha \beta} X_{\alpha \beta}\right)^{\frac{1}{2}}$. Since $\Phi$ attains its minimum on $\mathcal{B}$ and $\Psi>0$, we conclude that there exists $\alpha_{M}>0$ such that

$$
\Psi\left(\frac{X_{\alpha \beta}}{\|X\|}\right) \geq \alpha_{M}, \quad \text { for all symmetric tensor } X=\left(X_{\alpha \beta}\right) \not \equiv 0
$$

From the above inequality we deduce

$$
\bar{c}_{M}^{\alpha \beta \rho \sigma} X_{\alpha \beta} X_{\rho \sigma} \geq \alpha_{M} X_{\alpha \beta} X_{\alpha \beta}
$$

This means that the tensor $\left(\bar{c}_{M}^{\alpha \beta \sigma \tau}\right)$ is coercive. In a similar way we obtain that the tensors $\left(\bar{c}_{F}^{\alpha \beta \sigma \tau}\right)$ and $\left(\bar{d}^{\alpha \beta}\right)$ are coercive. Hence, by Lax-Milgram theorem we deduce that the global problem (34) has a unique solution. This completes the proof of Theorem 4.2 . 
Theorem 4.4. We have the following strong convergence for the gradients

$$
\left\{\begin{aligned}
\mathcal{T}^{\varepsilon}\left(\gamma_{\alpha \beta}\left(\boldsymbol{u}^{\varepsilon}\right)\right) \rightarrow \gamma_{\alpha \beta}(\boldsymbol{u})+s_{\alpha \beta, y}(\overline{\boldsymbol{u}}) & & \text { strongly in } L^{2}\left(\Omega \times Y^{*}\right), \\
\mathcal{T}^{\varepsilon}\left(\rho_{\alpha \beta}\left(\boldsymbol{u}^{\varepsilon}\right)\right) \rightarrow \rho_{\alpha \beta}(\boldsymbol{u})+r_{\alpha \beta, y}(\overline{\boldsymbol{u}}) & & \text { strongly in } \quad L^{2}\left(\Omega \times Y^{*}\right), \\
\mathcal{T}^{\varepsilon}\left(\nabla_{x} \varphi^{\varepsilon}\right) \rightarrow \nabla_{x} \varphi+\nabla_{y} \bar{\varphi} & \text { strongly in } & L^{2}\left(\Omega \times Y^{*} ; \mathbb{R}^{2}\right) .
\end{aligned}\right.
$$

Proof. Let us take $\boldsymbol{v}=\boldsymbol{u}, \psi=\varphi$ and $\overline{\boldsymbol{v}}=\overline{\boldsymbol{u}}, \bar{\psi}=\bar{\varphi}$ in (20). By summation we obtain

$$
\left\{\begin{array}{l}
\int_{\Omega \times Y^{*}}\left(c_{M}^{\alpha \beta \sigma \tau}\left(\gamma_{\alpha \beta}(\boldsymbol{u})+s_{\alpha \beta, y}(\overline{\boldsymbol{u}})\right)\left(\gamma_{\sigma \tau}(\boldsymbol{u})+s_{\sigma \tau, y}(\overline{\boldsymbol{u}})\right)\right. \\
\left.+\frac{t^{2}}{3} c_{F}^{\alpha \beta \sigma \tau}\left(\rho_{\alpha \beta}(\boldsymbol{u})+r_{\alpha \beta, y}(\overline{\boldsymbol{u}})\right)\left(\rho_{\sigma \tau}(\boldsymbol{u})+r_{\sigma \tau, y}(\overline{\boldsymbol{u}})\right)\right) \sqrt{a} \mathrm{~d} x \mathrm{~d} y \\
\left.+\int_{\Omega \times Y^{*}} d^{\alpha \beta}\left(\partial_{\alpha} \varphi+\partial_{\alpha, y} \bar{\varphi}\right)\left(\partial_{\beta} \psi+\partial_{\beta, y} \bar{\psi}\right)\right) \sqrt{a} \mathrm{~d} x \mathrm{~d} y=\left|Y^{*}\right| \int_{\Omega} \boldsymbol{p} \cdot \boldsymbol{v} \sqrt{a} \mathrm{~d} x
\end{array}\right.
$$

From (24) and (25), the left hand side in (46) is smaller than

$$
\begin{aligned}
& \liminf _{\varepsilon \rightarrow 0} \int_{\Omega \times Y^{*}}\left\{c_{M}^{\alpha \beta \sigma \tau} \mathcal{T}^{\varepsilon}\left(\gamma_{\alpha \beta}\left(\boldsymbol{u}^{\varepsilon}\right)\right) \mathcal{T}^{\varepsilon}\left(\gamma_{\sigma \tau}\left(\boldsymbol{u}^{\varepsilon}\right)\right)\right. \\
& \left.\quad+\frac{t^{2}}{3} c_{F}^{\alpha \beta \sigma \tau} \mathcal{T}^{\varepsilon}\left(\rho_{\alpha \beta}\left(\boldsymbol{u}^{\varepsilon}\right)\right) \mathcal{T}^{\varepsilon}\left(\rho_{\sigma \tau}\left(\boldsymbol{u}^{\varepsilon}\right)\right)+d^{\alpha \beta} \mathcal{T}^{\varepsilon}\left(\partial_{\alpha} \varphi^{\varepsilon}\right) \mathcal{T}^{\varepsilon}\left(\partial_{\beta} \varphi^{\varepsilon}\right)\right\} \mathcal{T}^{\varepsilon}(\sqrt{a}) \mathrm{d} x \mathrm{~d} y
\end{aligned}
$$

which reads

$$
\liminf _{\varepsilon \rightarrow 0} \int_{\Omega \times Y^{*}} \mathcal{T}^{\varepsilon}\left(\left\{c_{M}^{\alpha \beta \sigma \tau} \gamma_{\alpha \beta}\left(\boldsymbol{u}^{\varepsilon}\right) \gamma_{\sigma \tau}\left(\boldsymbol{u}^{\varepsilon}\right)+\frac{t 2}{3} c_{F}^{\alpha \beta \sigma \tau} \rho_{\alpha \beta}\left(\boldsymbol{u}^{\varepsilon}\right) \rho_{\sigma \tau}\left(\boldsymbol{u}^{\varepsilon}\right)+d^{\alpha \beta} \partial_{\alpha} \varphi^{\varepsilon} \partial_{\beta} \varphi^{\varepsilon}\right\} \sqrt{a}\right) \mathrm{d} x \mathrm{~d} y
$$

By virtue of Proposition $3.2(i)$ and (21), we have

$$
\begin{aligned}
& \liminf _{\varepsilon \rightarrow 0} \int_{\Omega \times Y^{*}} \mathcal{T}^{\varepsilon}\left(\left\{c_{M}^{\alpha \beta \sigma \tau} \gamma_{\alpha \beta}\left(\boldsymbol{u}^{\varepsilon}\right) \gamma_{\sigma \tau}\left(\boldsymbol{u}^{\varepsilon}\right)+\frac{t^{2}}{3} c_{F}^{\alpha \beta \sigma \tau} \rho_{\alpha \beta}\left(\boldsymbol{u}^{\varepsilon}\right) \rho_{\sigma \tau}\left(\boldsymbol{u}^{\varepsilon}\right)+d^{\alpha \beta} \partial_{\alpha} \varphi^{\varepsilon} \partial_{\beta} \varphi^{\varepsilon}\right\} \sqrt{a}\right) \mathrm{d} x \mathrm{~d} y \\
& \leq \liminf _{\varepsilon \rightarrow 0} \int_{\Omega^{\varepsilon}}\left(c_{M}^{\alpha \beta \sigma \tau} \gamma_{\alpha \beta}\left(\boldsymbol{u}^{\varepsilon}\right) \gamma_{\sigma \tau}\left(\boldsymbol{u}^{\varepsilon}\right)+\frac{t^{2}}{3} c_{F}^{\alpha \beta \sigma \tau} \rho_{\alpha \beta}\left(\boldsymbol{u}^{\varepsilon}\right) \rho_{\sigma \tau}\left(\boldsymbol{u}^{\varepsilon}\right)+d^{\alpha \beta} \partial_{\alpha} \varphi^{\varepsilon} \partial_{\beta} \varphi^{\varepsilon}\right) \sqrt{a} \mathrm{~d} x \\
& \leq \limsup _{\varepsilon \rightarrow 0} \int_{\Omega^{\varepsilon}}\left(c_{M}^{\alpha \beta \sigma \tau} \gamma_{\alpha \beta}\left(\boldsymbol{u}^{\varepsilon}\right) \gamma_{\sigma \tau}\left(\boldsymbol{u}^{\varepsilon}\right)+\frac{t^{2}}{3} c_{F}^{\alpha \beta \sigma \tau} \rho_{\alpha \beta}\left(\boldsymbol{u}^{\varepsilon}\right) \rho_{\sigma \tau}\left(\boldsymbol{u}^{\varepsilon}\right)+d^{\alpha \beta} \partial_{\alpha} \varphi^{\varepsilon} \partial_{\beta} \varphi^{\varepsilon}\right) \sqrt{a} \mathrm{~d} x \\
& =\limsup _{\varepsilon \rightarrow 0} \int_{\Omega^{\varepsilon}} \boldsymbol{p} \cdot \boldsymbol{u}^{\varepsilon} \sqrt{a} \mathrm{~d} x=\lim _{\varepsilon \rightarrow 0} \int_{\Omega^{\varepsilon}} \boldsymbol{p} \cdot \boldsymbol{u}^{\varepsilon} \sqrt{a} \mathrm{~d} x .
\end{aligned}
$$

Using (11), Proposition $3.2\left(\right.$ iii) and the fact that $\mathcal{T}^{\varepsilon}\left(\boldsymbol{u}^{\varepsilon}\right) \rightarrow \boldsymbol{u}$, we have

$$
\lim _{\varepsilon \rightarrow 0} \int_{\Omega^{\varepsilon}} \boldsymbol{p} \cdot \boldsymbol{u}^{\varepsilon} \sqrt{a} \mathrm{~d} x=\lim _{\varepsilon \rightarrow 0} \int_{\Omega^{\varepsilon} \times Y^{*}} \mathcal{T}^{\varepsilon}\left(\boldsymbol{u}^{\varepsilon}\right) \cdot \mathcal{T}^{\varepsilon}(\boldsymbol{p} \sqrt{a}) \mathrm{d} x \mathrm{~d} y=\left|Y^{*}\right| \int_{\Omega} \boldsymbol{p} \cdot \boldsymbol{u} \sqrt{a} \mathrm{~d} x .
$$

From (46) and (47) we deduce that all the above inequalities become equalities. This also implies the strong convergences (45). The proof of Theorem 4.4 is now complete.

\section{Conclusions}

We have obtained in this paper the limit constitutive law of a piezoelectric material with periodically perforated microstructure and whose reference configuration is a thin shell with fixed thickness. The main difficulty 
comes from the geometry of the reference cell which presents holes. Furthermore, the local problems for the shells are of different nature than the global one, due to the different orders of derivatives in the linearized change of metric and curvature tensors $\gamma$ and $\rho$. An interesting direction for further research is to determine the asymptotic behavior of the displacement elastic field and of the electric potential when both $\varepsilon$ and the thickness

of the shell $2 t$ goes to zero. In this case the ratio $\frac{\varepsilon}{t}$ plays an important role as it was analyzed in Caillerie and Sanchez-Palencia [6].

\section{Appendix. Korn's INEQUALity FOR SHELls In PERFORATED DOMAINS}

In this section we prove the Korn's inequality for perforated domains. Let $D=] 0, L[\times \mathbb{R}, L>0$.

Lemma 6.1. Let $V \in H^{1}(D)$ be such that $V(0, \cdot)=V(L, \cdot)=0$. We extend $V$ by zero outside $D$ and let $\Lambda: \mathbb{Z}^{2} \rightarrow(0,+\infty)$ with the property

$$
\|\nabla V\|_{L^{2}\left(\varepsilon(\xi+Y) ; \mathbb{R}^{2}\right)}^{2} \leq \Lambda(\xi)+c\|V\|_{L^{2}(\varepsilon(\xi+Y))}^{2},
$$

for all $\xi \in \mathbb{Z}^{2}$, where $c>0$ is a positive constant that does not depend on $\varepsilon$ and satisfies $c \varepsilon L \leq 1 / 2$. Then, there exists $C>0$ not depending on $\varepsilon$ such that

$$
\|V\|_{H^{1}(D)}^{2} \leq C \sum_{\xi \in \mathbb{Z}^{2}} \Lambda(\xi) .
$$

Proof. Since $V(0, \cdot)=0$, for all $k \in \mathbb{Z}, k \geq 0$ we have

$$
\|V\|_{L^{2}(\varepsilon((k, q)+Y))}^{2} \leq \varepsilon L \sum_{p=0}^{k}\|\nabla V\|_{L^{2}\left(\varepsilon((p, q)+Y) ; \mathbb{R}^{2}\right)}^{2} .
$$

We fix $q \in \mathbb{Z}, q \geq 0$ and set

$$
y_{k}=\sum_{p=0}^{k}\|\nabla V\|_{L^{2}\left(\varepsilon((p, q)+Y) ; \mathbb{R}^{2}\right)}^{2} .
$$

From (48) and (50) it follows that

$$
y_{0} \leq 2 \Lambda(0, q)
$$

and

$$
y_{k+1}-y_{k} \leq \Lambda(k+1, q)+c L \varepsilon y_{k+1} .
$$

Since $2 c \varepsilon L \leq 1$, the last inequality yields

$$
y_{k+1}-y_{k} \leq 2 \Lambda(k+1, q)+2 c L \varepsilon y_{k} .
$$

Let $K_{\varepsilon}=\left[\frac{L}{\varepsilon}\right]$. For all $p>K_{\varepsilon}+1$ we have $V=0$ in $L^{2}(\varepsilon((p, q)+Y))$, hence one can assume that $k \leq K_{\varepsilon}+1$. Summating in (52) we obtain

$$
y_{k} \leq(1+2 c \varepsilon L)^{k} \sum_{p=1}^{k} \Lambda(p, q)(1+2 c \varepsilon L)^{-p}+y_{0}(1+2 c \varepsilon L)^{k} .
$$

Using (51) we deduce

$$
y_{k} \leq 2(1+2 c \varepsilon L)^{k} \sum_{p=0}^{k} \Lambda(p, q)(1+2 c \varepsilon L)^{-p} \leq 2(1+2 c \varepsilon L)^{k} \sum_{p=0}^{k} \Lambda(p, q) .
$$


Since $k \leq\left[\frac{L}{\varepsilon}\right]+1$ we also have $(1+2 c \varepsilon L)^{k} \leq e^{2 c \varepsilon L k} \leq e^{2 c L 2+1}$. This yields

$$
y_{k} \leq 2 e^{2 c L 2+1} \sum_{p=0}^{k} \Lambda(p, q)
$$

Summating over $q$ in the above inequality we get

$$
\|\nabla V\|_{L^{2}\left(D ; \mathbb{R}^{2}\right)}^{2} \leq C \sum_{(p, q) \in \mathbb{Z}^{2}} \Lambda(p, q)
$$

Now, (49) follows from the last relation and Poincaré's inequality. This concludes the proof.

On $\boldsymbol{V}\left(\Omega^{\varepsilon}\right)$ we define the seminorm

$$
\|\boldsymbol{v}\| \|_{\varepsilon}:=\left(\sum_{\alpha, \beta}\left\{\left\|\rho_{\alpha \beta}(\boldsymbol{v})\right\|_{L^{2}\left(\Omega^{\varepsilon}\right)}^{2}+\left\|\gamma_{\alpha \beta}(\boldsymbol{v})\right\|_{L^{2}\left(\Omega^{\varepsilon}\right)}^{2}\right\}\right)^{1 / 2}, \quad \text { for all } \boldsymbol{v} \in \boldsymbol{V}\left(\Omega^{\varepsilon}\right)
$$

The following result asserts that $\||\cdot|\|_{\varepsilon}$ is equivalent to the standard norm in $H^{1}\left(\Omega^{\varepsilon}\right) \times H^{1}\left(\Omega^{\varepsilon}\right) \times H^{2}\left(\Omega^{\varepsilon}\right)$.

Theorem 6.2 (Korn's inequality). On $\boldsymbol{V}\left(\Omega^{\varepsilon}\right)$, the seminorm $\|\cdot\| \|_{\varepsilon}$ is equivalent to the usual norm in $H^{1}\left(\Omega^{\varepsilon}\right) \times$ $H^{1}\left(\Omega^{\varepsilon}\right) \times H^{2}\left(\Omega^{\varepsilon}\right)$. More precisely, there exist two positive constants $c_{1}, c_{2}>0$ depending only on $\Omega$, $Y^{*}$ and on the mapping $\theta$ (but not depending on $\varepsilon$ ) such that

$$
c_{1}\|\| \boldsymbol{v}\|\|_{\varepsilon} \leq\left\|v_{1}\right\|_{H^{1}\left(\Omega^{\varepsilon}\right)}+\left\|v_{2}\right\|_{H^{1}\left(\Omega^{\varepsilon}\right)}+\left\|v_{3}\right\|_{H^{2}\left(\Omega^{\varepsilon}\right)} \leq c_{2}\|\| \boldsymbol{v}\|\|_{\varepsilon}, \quad \text { for all } \boldsymbol{v} \in \boldsymbol{V}\left(\Omega^{\varepsilon}\right)
$$

Proof. The existence of the constant $c_{1}>0$ in (53) is obvious.

Since $\partial Y^{*}$ has a Lipschitz boundary, there exists a linear and continuous operator $\mathcal{P}: H^{1}\left(Y^{*}\right) \rightarrow H^{1}(Y)$ such that for all $\psi \in H^{1}\left(Y^{*}\right)$ we have $\mathcal{P}(\psi)=\psi$ in $Y^{*}$ and

$$
\|\mathcal{P}(\psi)\|_{L^{2}(Y)} \leq \widetilde{C}\|\psi\|_{L^{2}\left(Y^{*}\right)}, \quad\left\|\nabla_{y} \mathcal{P}(\psi)\right\|_{L^{2}\left(Y ; \mathbb{R}^{2}\right)} \leq \widetilde{C}\left\|\nabla_{y} \psi\right\|_{L^{2}\left(Y^{*} ; \mathbb{R}^{2}\right)}
$$

for some constant $\tilde{C}>0$ that depends only on $\partial Y^{*}$. Hence, for all $u \in V\left(\Omega^{\varepsilon}\right)$ extended by zero on $\widetilde{\Omega}^{\varepsilon} \backslash \Omega^{\varepsilon}$, there exists a function $U \in H_{0}^{1}\left(\widetilde{\Omega}^{\varepsilon}\right)$ such that $U=u$ in $\Omega^{\varepsilon}$ and $\left\|\nabla_{x} U\right\|_{L^{2}\left(\widetilde{\Omega}^{\varepsilon} ; \mathbb{R}^{2}\right)} \leq C\left\|\nabla_{x} u\right\|_{L^{2}\left(\Omega^{\varepsilon} ; \mathbb{R}^{2}\right)}$, where $C>0$ does not depend on $\varepsilon$.

For $\boldsymbol{v} \in \boldsymbol{V}\left(\Omega^{\varepsilon}\right)$, let $V_{i}$ and $W_{\alpha}$ be the extensions to $H_{0}^{1}\left(\widetilde{\Omega}^{\varepsilon}\right)$ of $v_{i}$ and $\partial_{\alpha} v_{3}$ defined as above. Set $X=$ $\left(V_{1}, V_{2}, V_{3}, W_{1}, W_{2}\right) \in H_{0}^{1}\left(\widetilde{\Omega}^{\varepsilon} ; \mathbb{R}^{5}\right)$. In each cell $\varepsilon\left(\xi+Y^{*}\right) \subseteq \widetilde{\Omega}^{\varepsilon}, \xi \in \mathbb{Z}^{2}$, we have

$$
\begin{gathered}
\sum_{i}\left\|\nabla_{x} v_{i}\right\|_{L^{2}\left(\varepsilon\left(\xi+Y^{*}\right) ; \mathbb{R}^{2}\right)}^{2} \leq C\left(\sum_{\alpha, \beta}\left\|\gamma_{\alpha \beta}(\boldsymbol{v})\right\|_{L^{2}\left(\varepsilon\left(\xi+Y^{*}\right)\right)}^{2}+\|\boldsymbol{v}\|_{L^{2}\left(\varepsilon\left(\xi+Y^{*}\right) ; \mathbb{R}^{3}\right)}^{2}\right) \\
\sum_{\alpha}\left\|\nabla_{x}\left(\partial_{\alpha} v_{3}\right)\right\|_{L^{2}\left(\varepsilon\left(\xi+Y^{*}\right) ; \mathbb{R}^{2}\right)}^{2} \leq C \sum_{\alpha, \beta}\left\|\rho_{\alpha \beta}(\boldsymbol{v})\right\|_{L^{2}\left(\varepsilon\left(\xi+Y^{*}\right)\right)}^{2}+C\left(\left\|\nabla_{x} \boldsymbol{v}\right\|_{L^{2}\left(\varepsilon\left(\xi+Y^{*}\right) ; \mathbb{R}^{3} \times \mathbb{R}^{3}\right)}^{2}+\|\boldsymbol{v}\|_{L^{2}\left(\varepsilon\left(\xi+Y^{*}\right) ; \mathbb{R}^{3}\right)}^{2}\right),
\end{gathered}
$$

where $C>0$ does not depend on $\varepsilon$. This implies

$$
\left\|\nabla_{x} X\right\|_{L^{2}\left(\varepsilon\left(\xi+Y^{*}\right) ; \mathbb{R}^{5} \times \mathbb{R}^{5}\right)}^{2} \leq C_{1} \sum_{\alpha, \beta}\left\{\left\|\rho_{\alpha \beta}(\boldsymbol{v})\right\|_{L^{2}\left(\varepsilon\left(\xi+Y^{*}\right)\right)}^{2}+\left\|\gamma_{\alpha \beta}(\boldsymbol{v})\right\|_{L^{2}\left(\varepsilon\left(\xi+Y^{*}\right)\right)}^{2}\right\}+C_{1}\|X\|_{L^{2}\left(\varepsilon\left(\xi+Y^{*}\right) ; \mathbb{R}^{5}\right)}^{2}
$$


By virtue of (54) we deduce

$$
\left\|\nabla_{x} X\right\|_{L^{2}\left(\varepsilon(\xi+Y) ; \mathbb{R}^{5} \times \mathbb{R}^{5}\right)}^{2} \leq C_{2} \sum_{\alpha, \beta}\left\{\left\|\rho_{\alpha \beta}(\boldsymbol{v})\right\|_{L^{2}\left(\varepsilon\left(\xi+Y^{*}\right)\right)}^{2}+\left\|\gamma_{\alpha \beta}(\boldsymbol{v})\right\|_{L^{2}\left(\varepsilon\left(\xi+Y^{*}\right)\right)}^{2}\right\}+C_{2}\|X\|_{L^{2}\left(\varepsilon(\xi+Y) ; \mathbb{R}^{5}\right)}^{2},
$$

for some $C_{2}>0$ independent on $\varepsilon$.

Since $\Omega$ is bounded, without loosing the generality, one can assume that $\widetilde{\Omega}^{\varepsilon} \subset\left\{x \in \mathbb{R}^{2} ; 0<x_{1}<L\right\}$, for some $L>0$. In view of Lemma 6.1 it follows that

$$
\|X\|_{H^{1}\left(\Omega ; \mathbb{R}^{5}\right)}^{2} \leq C_{3} \sum_{\alpha, \beta}\left\{\left\|\rho_{\alpha \beta}(\boldsymbol{v})\right\|_{L^{2}\left(\Omega^{\varepsilon}\right)}^{2}+\left\|\gamma_{\alpha \beta}(\boldsymbol{v})\right\|_{L^{2}\left(\Omega^{\varepsilon}\right)}^{2}\right\}
$$

Moreover, since $V_{i}=v_{i}$ and $W_{\alpha}=\partial_{\alpha} v_{3}$ in $\Omega^{\varepsilon}$, from (55) we deduce

$$
\left\|v_{1}\right\|_{H^{1}\left(\Omega^{\varepsilon}\right)}^{2}+\left\|v_{2}\right\|_{H^{1}\left(\Omega^{\varepsilon}\right)}^{2}+\left\|v_{3}\right\|_{H^{2}\left(\Omega^{\varepsilon}\right)}^{2} \leq C_{3} \sum_{\alpha, \beta}\left\{\left\|\rho_{\alpha \beta}(\boldsymbol{v})\right\|_{L^{2}\left(\Omega^{\varepsilon}\right)}^{2}+\left\|\gamma_{\alpha \beta}(\boldsymbol{v})\right\|_{L^{2}\left(\Omega^{\varepsilon}\right)}^{2}\right\}
$$

This completes the proof.

Acknowledgements. This work has been supported by the European Programs "Smart System" HPRN-CT-2002-00284 and "Some nonclassical problems for thin structure" INTAS 06-1000017-8886.

\section{REFERENCES}

[1] G. Allaire, Homogenization and two-scale convergence. SIAM J. Math. Anal. 23 (1992) 1482-1518.

[2] T. Arbogast, J. Douglas and U. Hornung, Derivation of the double porosity model of single phase flow in homogenization theory. SIAM J. Math. Anal. 21 (1990) 823-836.

[3] A. Bensoussan, J.-L. Lions and G. Papanicolaou, Asymptotic methods in periodic media. North Holland (1978).

[4] A. Bourgeat, J.B. Castillero, J.A. Otero and R.R. Ramos, Asymptotic homogenization of laminated piezocomposite materials. Int. J. Solids Structures 35 (1998) 527-541.

[5] D. Caillerie and E. Sanchez-Palencia, A new kind of singular stiff problems and application to thin elastic shells. Math. Models Methods Appl. Sci. 5 (1995) 47-66.

[6] D. Caillerie and E. Sanchez-Palencia, Elastic thin shells: Asymptotic theory in the anisotropic and heterogeneous cases. Math. Models Methods Appl. Sci. 5 (1995) 473-496.

[7] A. Cioranescu, A. Damlamian and G. Griso, Periodic unfolding and homogenization. C. R. Acad. Sci. Paris, Sér. I 335 (2002) 99-104.

[8] D. Cioranescu and P. Donato, An introduction to homogenization. Oxford University Press (1999).

[9] D. Cioranescu and P. Donato, The periodic unfolding method in perforated domains,Portugaliae Mathematica, Vol. 63, Fasc. 4 (2006) 467-496.

[10] D. Cioranescu and J. Saint-Jean Paulin, Homogenization of reticulated structures. Springer-Verlag, New-York (1999).

[11] D. Cioranescu, P. Donato and R. Zaki, The periodic unfolding method in perforated domains. Porth. Math. N.S. 63 (2006) 467-496.

[12] E. Dieulesaint and D. Royer, Ondes élastiques dans les solides, application au traitement du signal. Masson, Paris (1974).

[13] C. Haenel, Analyse et simulation numérique de coques piézoélectriques. Ph.D. thesis, Université Pierre et Marie Curie, France (2000).

[14] T. Ikeda, Fundamentals of piezoelectricity. Oxford University Press (1990).

[15] W.T. Koiter, On the foundations of the linear theory of thin elastic shell. Proc. Kon. Ned. Akad. Wetensch. B73 (1970) $169-195$. 
[16] T. Lewiński and J.J. Telega, Plates, laminates and shells. Asymptotic analysis and homogenization, Advances in Mathematics for Applied Sciences. World Scientific (2000).

[17] S. Luckhaus, A. Bourgeat and A. Mikelic, Convergence of the homogenization process for a double porosity model of immiscible two phase flow. SIAM J. Math. Anal. 27 (1996) 1520-1543.

[18] H. Mechkour, Homogénéisation et simulation numérique de structures piézoeléctriques perforées et laminées. Ph.D. thesis, ESIEE-Paris (2004).

[19] B. Miara, E. Rohan, M. Zidi and B. Labat, Piezomaterials for bone regeneration design. Homogenization approach. J. Mech. Phys. Solids 53 (2005) 2529-2556.

[20] G. Nguetseng, A general convergence result for a functional related to the theory of homogenisation. SIAM J. Math. Anal. 20 (1989) 608-623.

[21] A. Preumont, A. François and P. de Man, Spatial filtering with piezoelectric films via porous electrod design, in Proc. of 13th Int. Conf. on Adaptive Structures and Technologies, Berlin (2002).

[22] J. Sanchez-Hubert and E. Sanchez-Palencia, Introduction aux méthodes asymptotiques et à l'homogénéisation. Application à la Mécanique des milieux continus. Masson, Paris (1992).

[23] J. Sanchez-Hubert and E. Sanchez-Palencia, Coques élastiques minces. Propriétés asymptotiques. Masson, Paris (1997). 